\title{
Synthesis and Characterization of Cruciform-conjugated Molecules Based on Tetrathiafulvalene
}

Mikkel Vestergaard, Karsten Jennum, Jakob Kryger Sørensen, Kristine Kilså, Mogens Brøndsted Nielsen*

Department of Chemistry, University of Copenhagen, Universitetsparken 5, DK-2100 Copenhagen Ø, Denmark. E-mail: mbn@kiku.dk

\section{Table of Contents:}

General Experimental Methods:

Page

NMR spectra of compounds 3-5, 8-11, 13-18:

S2

Calculations: Atom Coordinates and Absolute Energies:

S3-27

S28-34 


\section{General Experimental Methods}

THF was distilled from $\mathrm{Na}$. $\mathrm{CH}_{2} \mathrm{Cl}_{2}$ and $\mathrm{MeCN}$ were both dried over molecular sieves (4 $\AA)$. Hay catalyst refers to $\mathrm{CuCl}(0.13 \mathrm{~g}, 1.3 \mathrm{mmol})$ and TMEDA $(0.16 \mathrm{~g}, 1.4 \mathrm{mmol})$ in $\mathrm{CH}_{2} \mathrm{Cl}_{2}(5.0 \mathrm{~mL})$. All reactions, except for the oxidative Hay couplings, were carried out under an atmosphere of Ar. Thin-layer chromatography (TLC) was carried out using aluminum sheets pre-coated with silica gel. Column chromatography was carried out using silica gel $(0.040-0.063 \mathrm{~mm})$. Melting points are uncorrected. Fast atom bombardment (FAB) spectra were obtained in the positive ion mode using 3-nitrobenzyl alcohol (NBA) as matrix. Cyclic voltammetry and differential pulse voltammetry were measured using a glassy carbon working electrode and a Pt wire counter electrode. All potentials are expressed relative to that of $\mathrm{Fc}^{+} / \mathrm{Fc}(0.31 \mathrm{~V}$ vs SCE; Bard, A. J.; Faulkner, L. R. Electrochemical Methods: Fundamentals and Applications, 2nd edition, John Wiley \& Sons, 2001) and were measured in $\mathrm{CH}_{2} \mathrm{Cl}_{2}$ with $0.1 \mathrm{M} \mathrm{Bu}_{4} \mathrm{NPF}_{6}$ as supporting electrolyte; scan rate $0.1 \mathrm{Vs}^{-1}$. 
${ }^{1} \mathrm{H}$ NMR Spectrum (300 $\left.\mathrm{MHz}, \mathrm{CDCl}_{3}\right)$ of compound 8
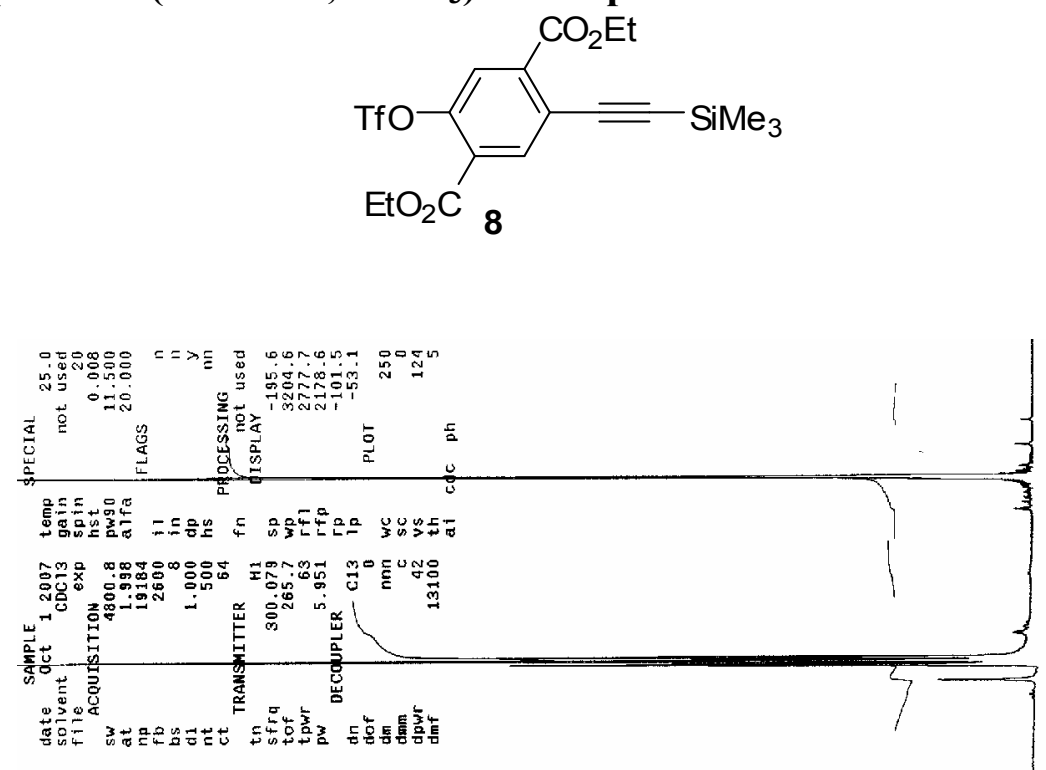

흠

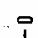

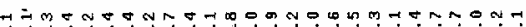

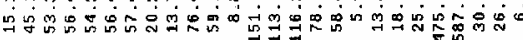

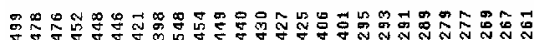

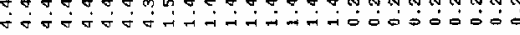

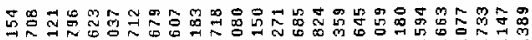

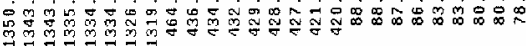

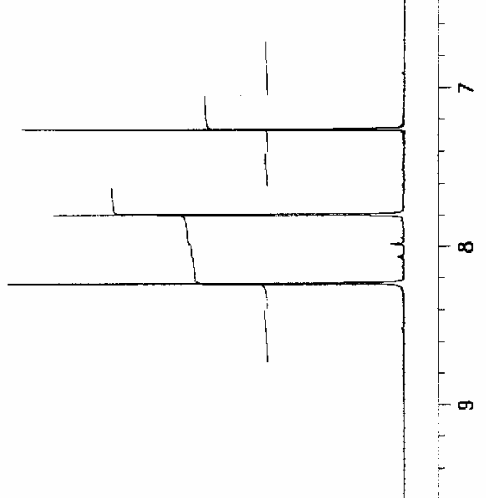


${ }^{13} \mathrm{C}$ NMR Spectrum (75 MHz, $\mathrm{CDCl}_{3}$ ) of compound 8
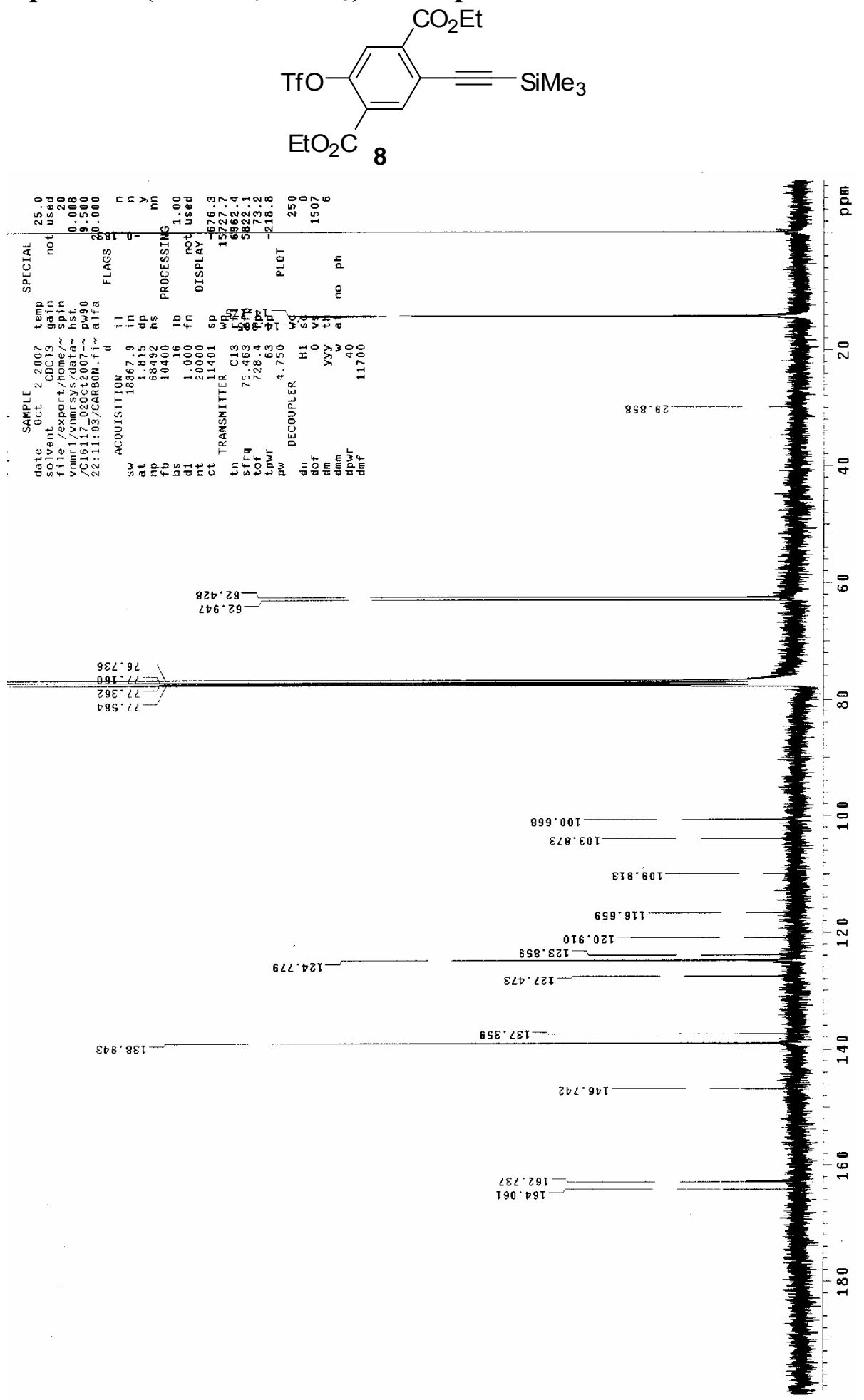
${ }^{1} \mathrm{H}$ NMR Spectrum (300 MHz, $\left.\mathrm{CDCl}_{3}\right)$ of compound 9
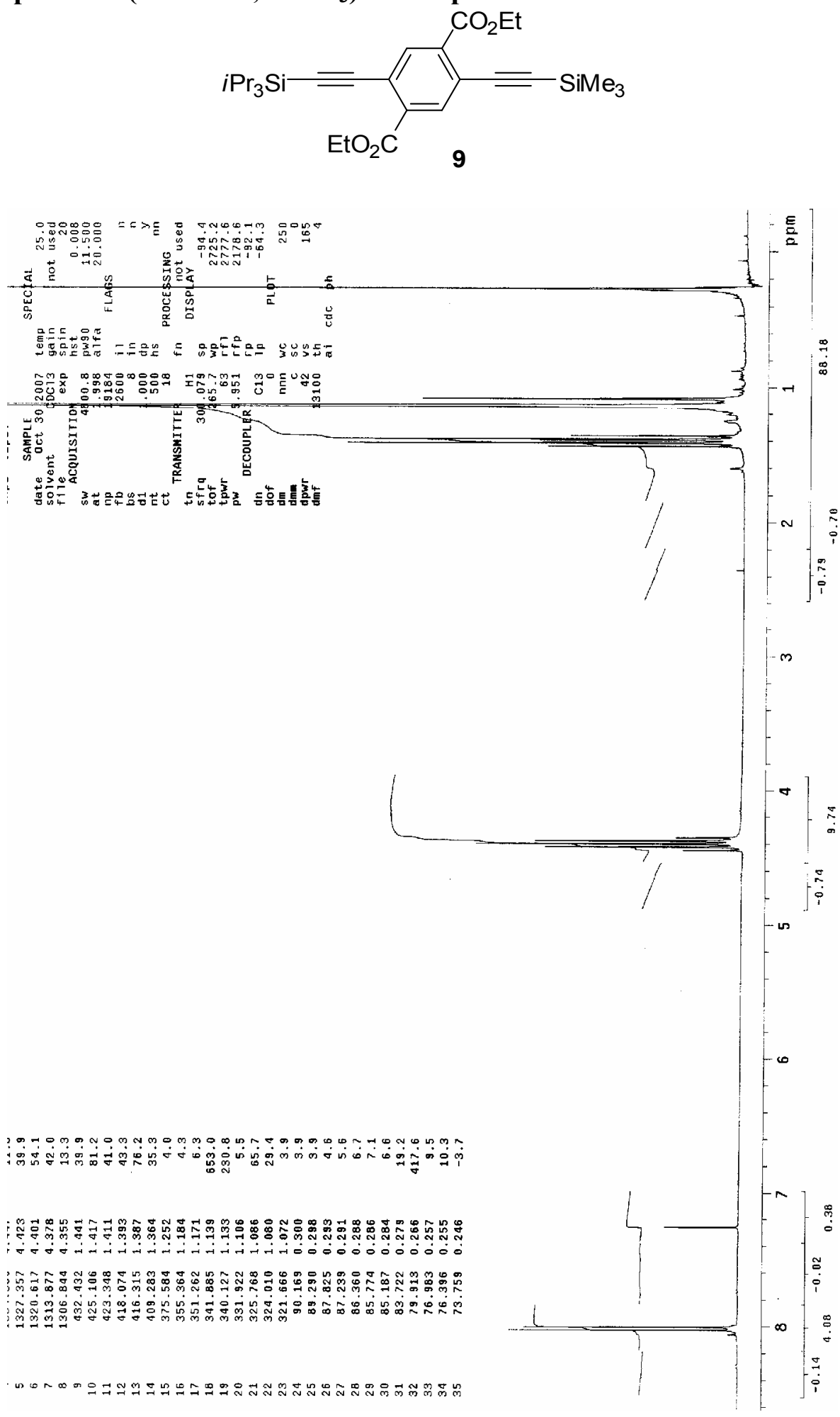


\section{${ }^{13} \mathrm{C}$ NMR Spectrum (75 MHz, $\mathrm{CDCl}_{3}$ ) of compound 9}
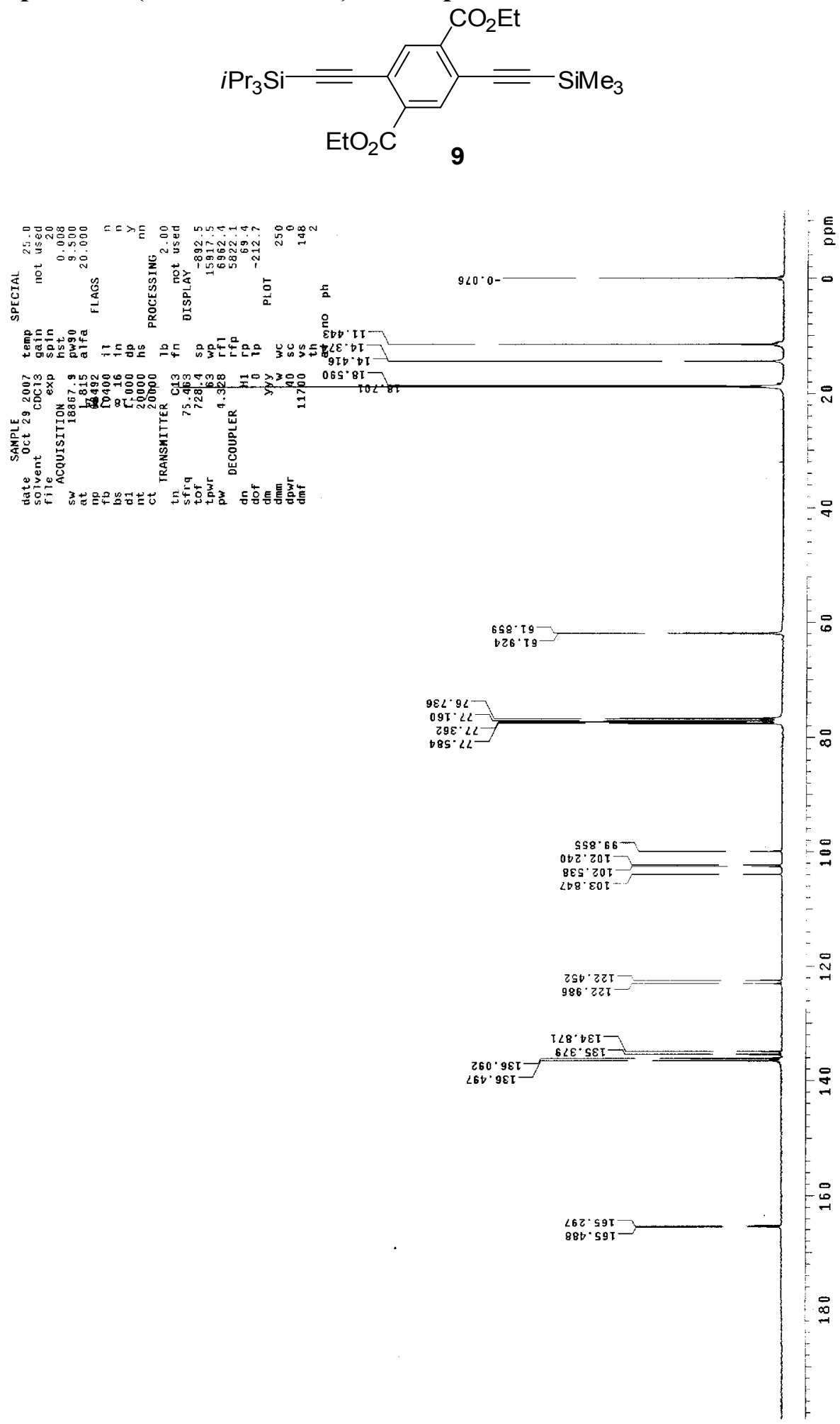
${ }^{1} \mathrm{H}$ NMR Spectrum (300 $\mathrm{MHz}, \mathrm{CDCl}_{3}$ ) of compound 10
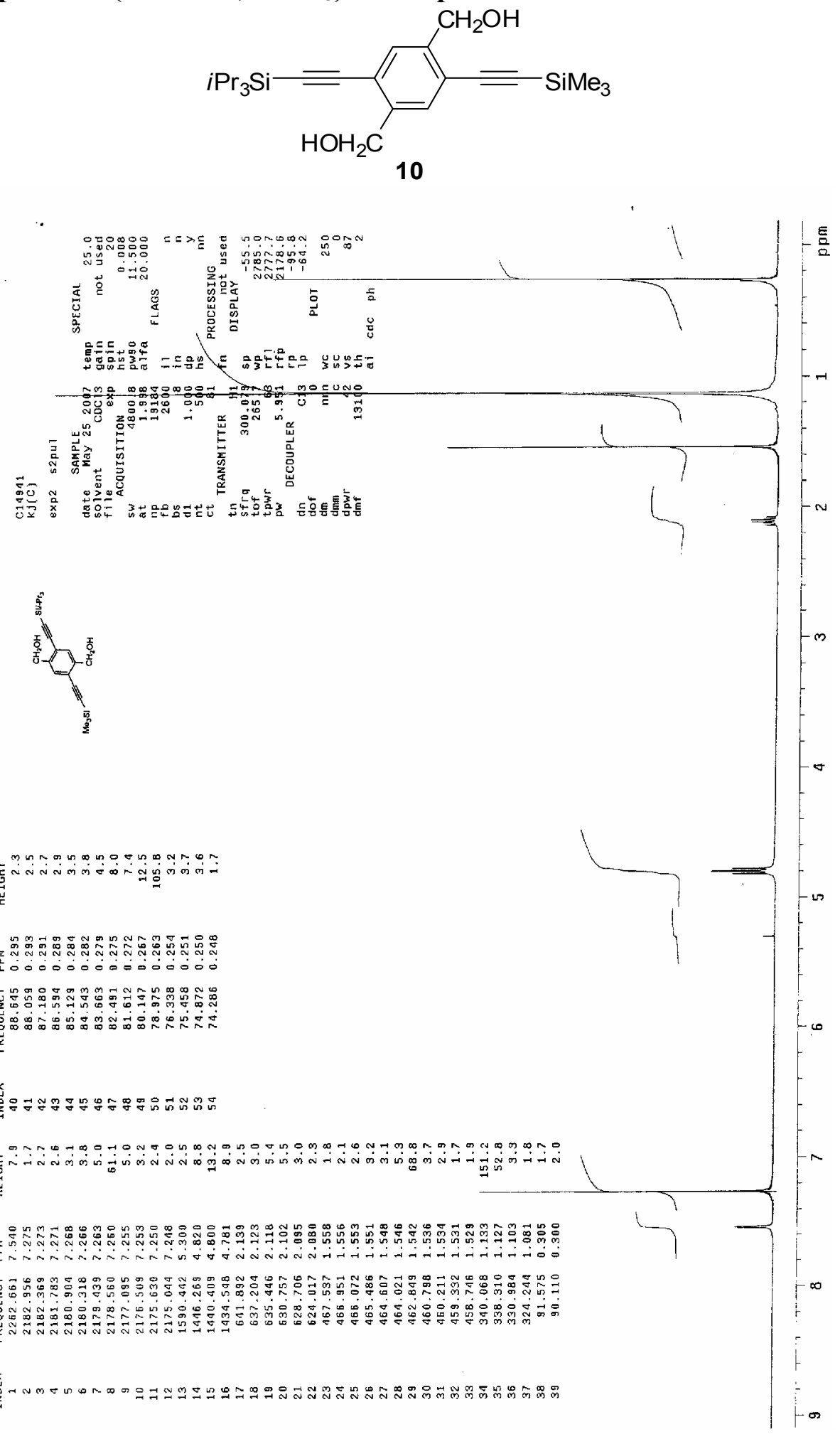
${ }^{13} \mathrm{C}$ NMR Spectrum (75 MHz, $\mathrm{CDCl}_{3}$ ) of compound 10
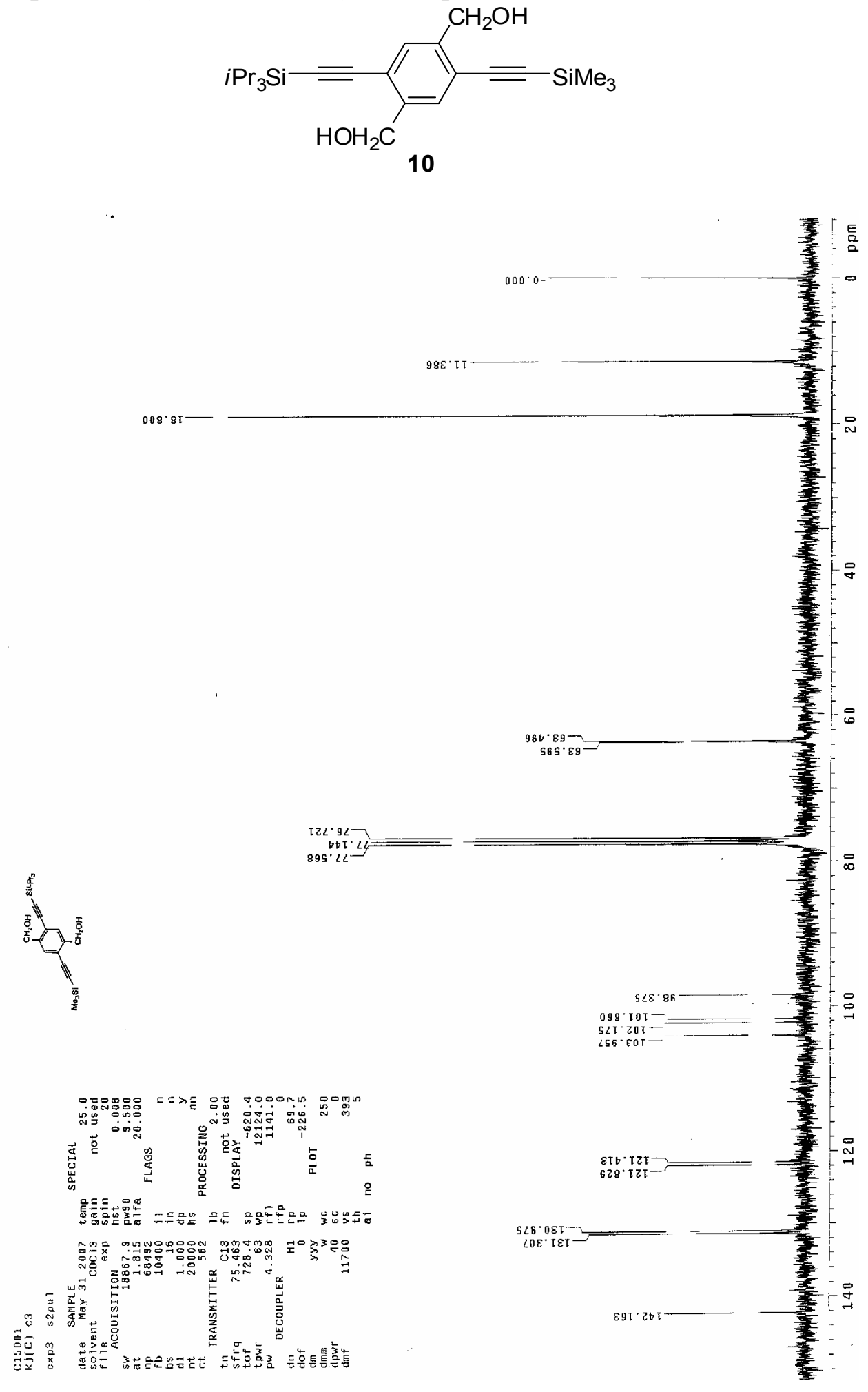
${ }^{1} \mathrm{H}$ NMR Spectrum (300 MHz, $\mathrm{CDCl}_{3}$ ) of compound 11

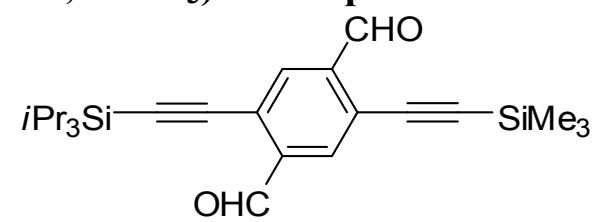

11

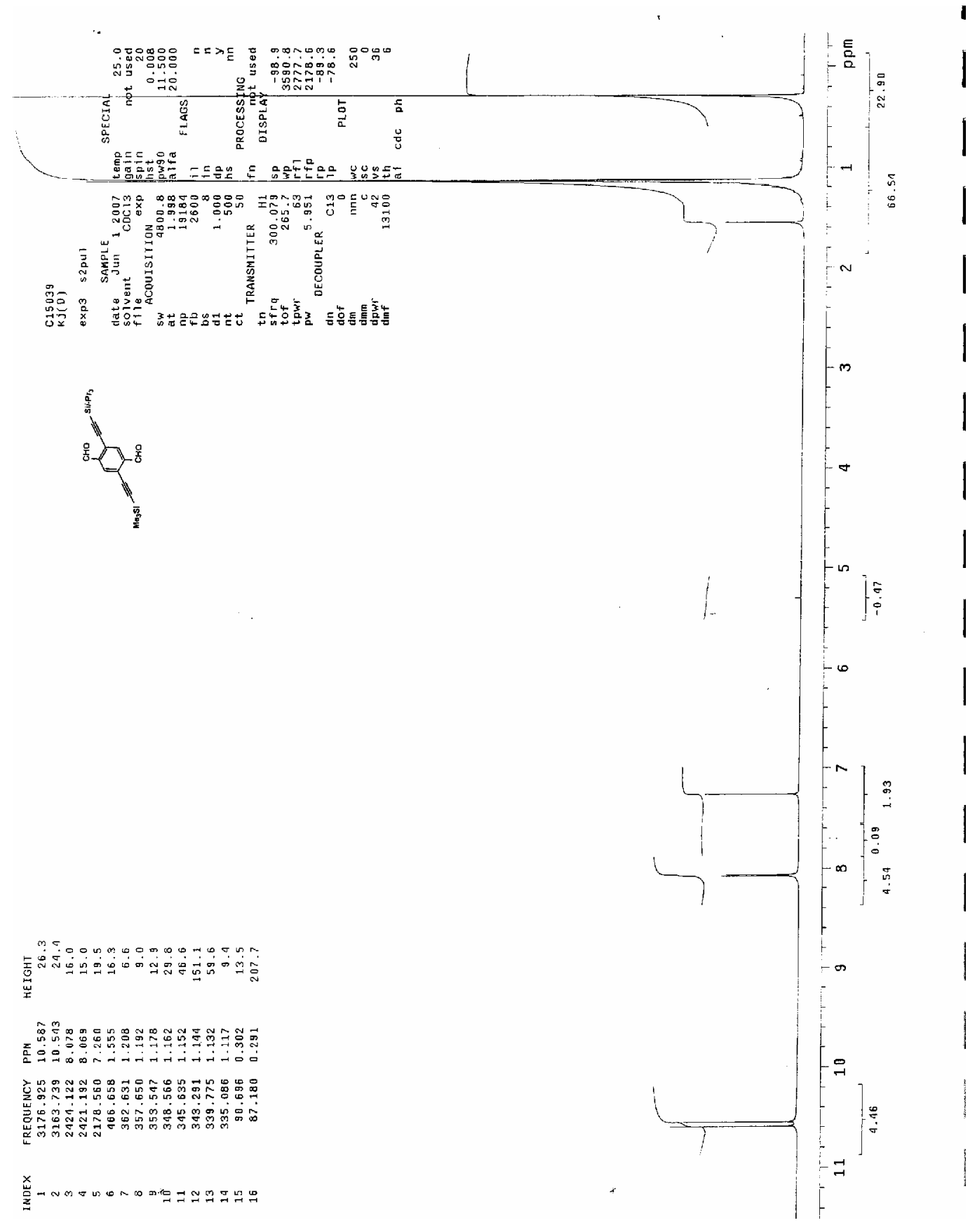




\section{${ }^{13} \mathrm{C}$ NMR Spectrum $\left(75 \mathrm{MHz}, \mathrm{CDCl}_{3}\right.$ ) of compound 11}

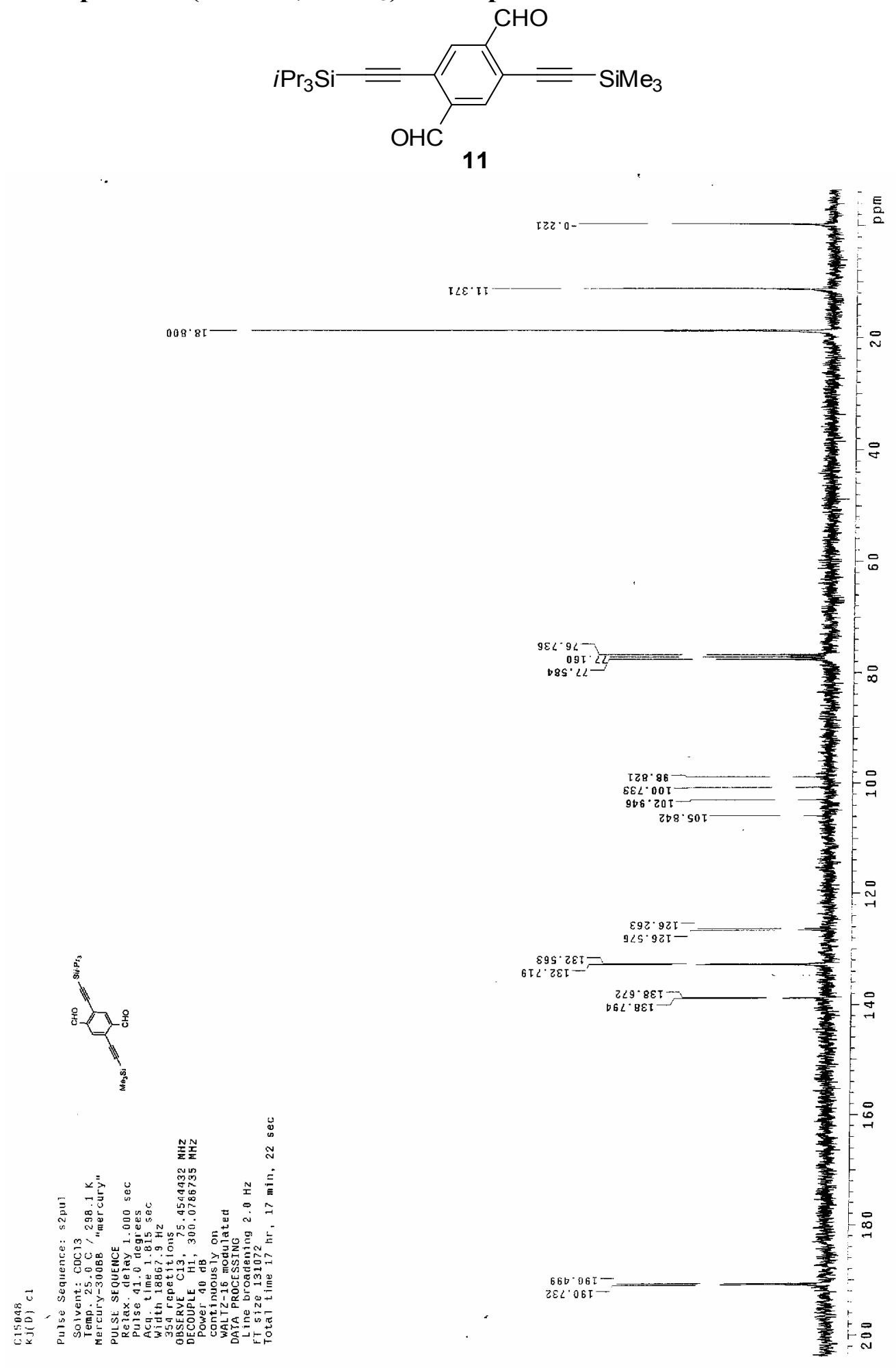


${ }^{1} \mathrm{H}$ NMR Spectrum (300 MHz, $\mathrm{CDCl}_{3}$ ) of compound 3
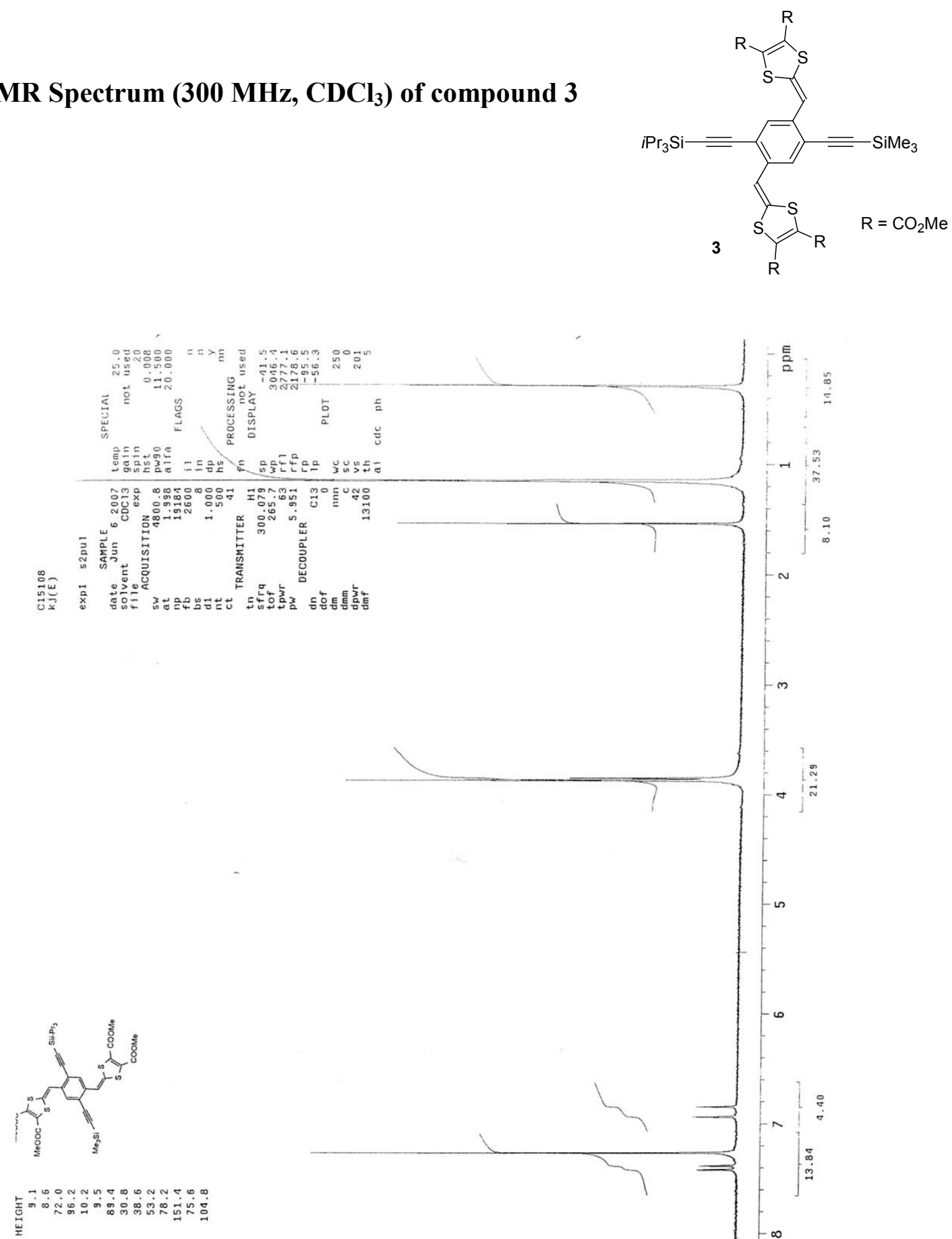

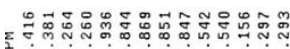

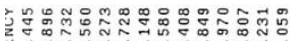

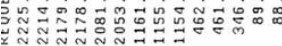

竎

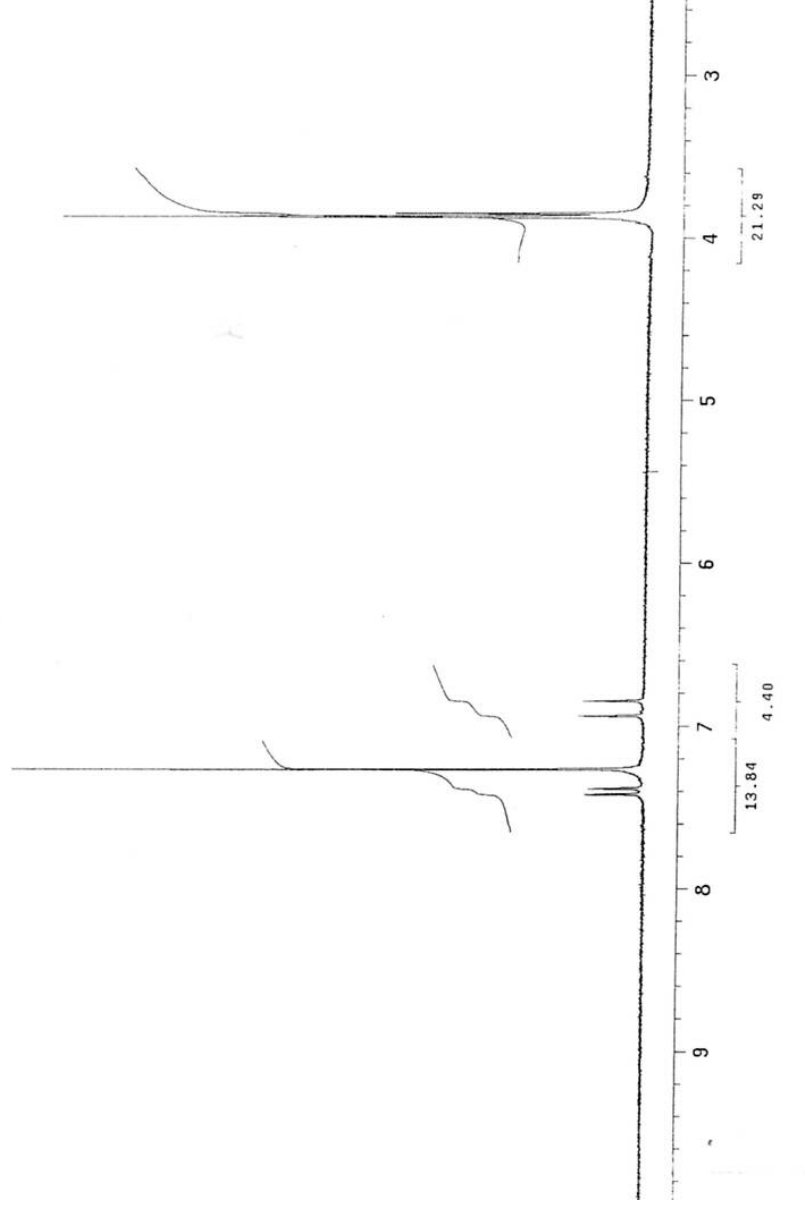


${ }^{13} \mathrm{C}$ NMR Spectrum (75 MHz, $\mathrm{CDCl}_{3}$ ) of compound 3
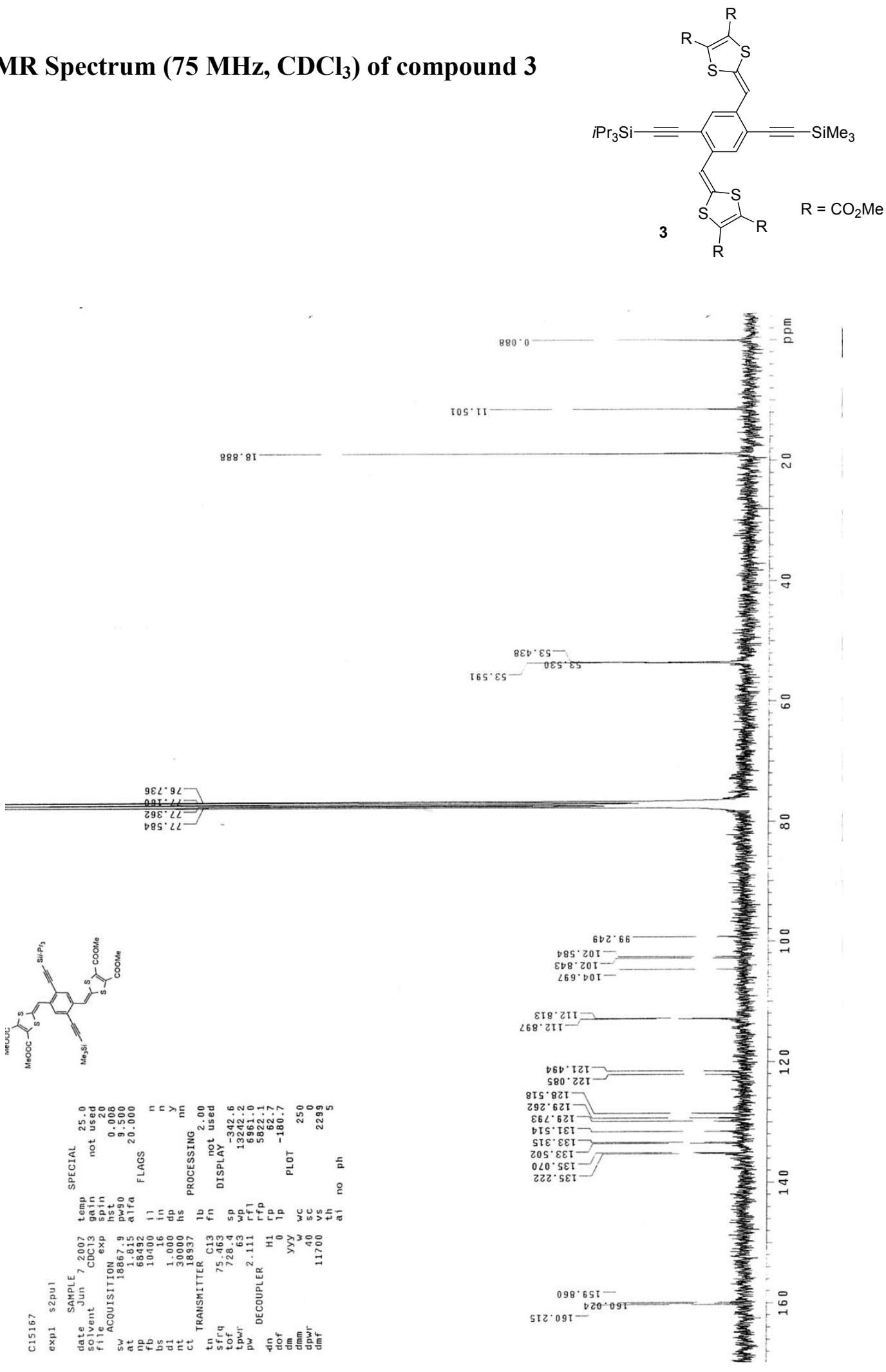
${ }^{1} \mathrm{H}$ NMR Spectrum (300 MHz, $\mathrm{CDCl}_{3}$ ) of compound 4

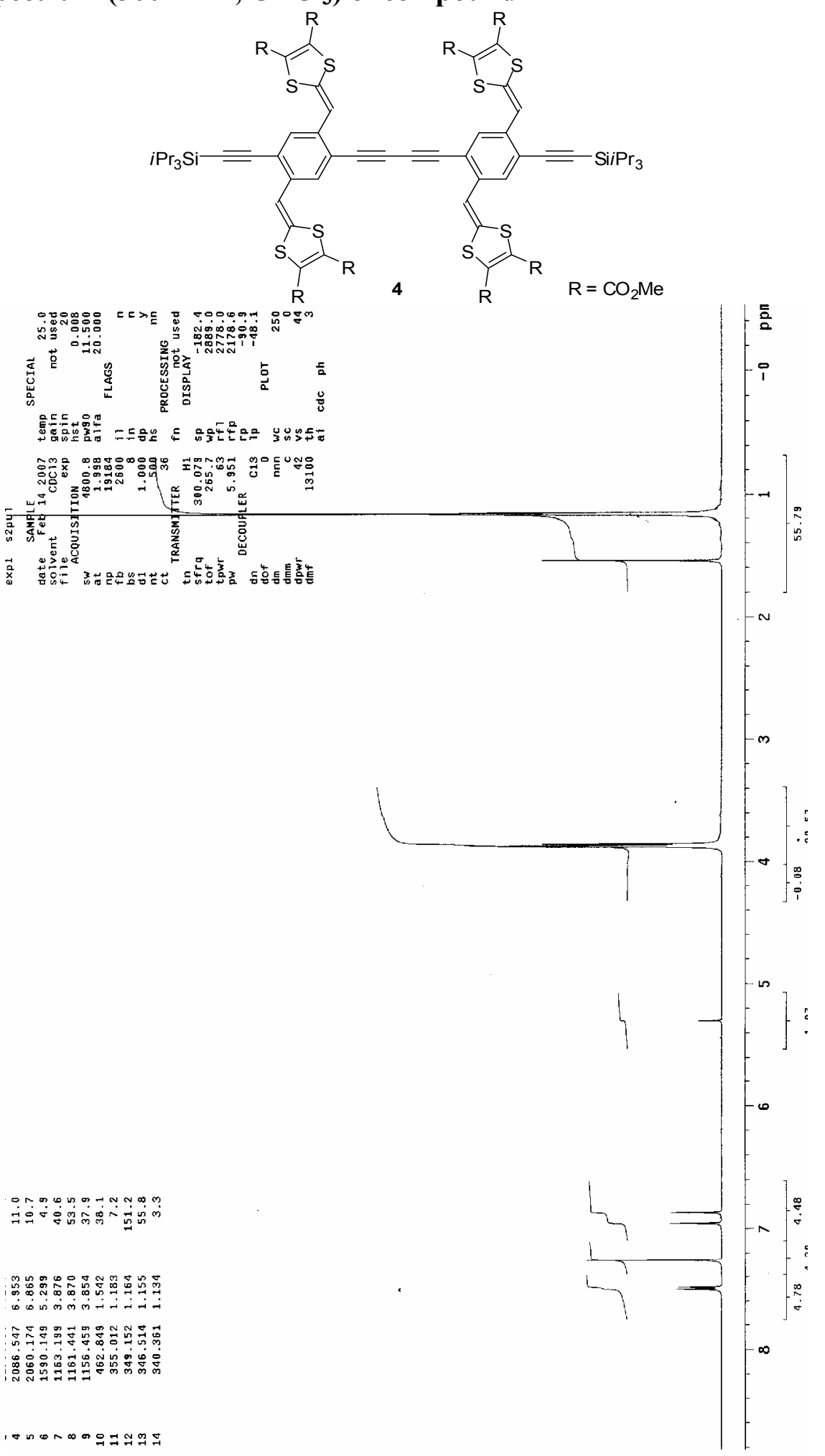


${ }^{13} \mathrm{C}$ NMR Spectrum (75 MHz, $\mathrm{CDCl}_{3}$ ) of compound 4
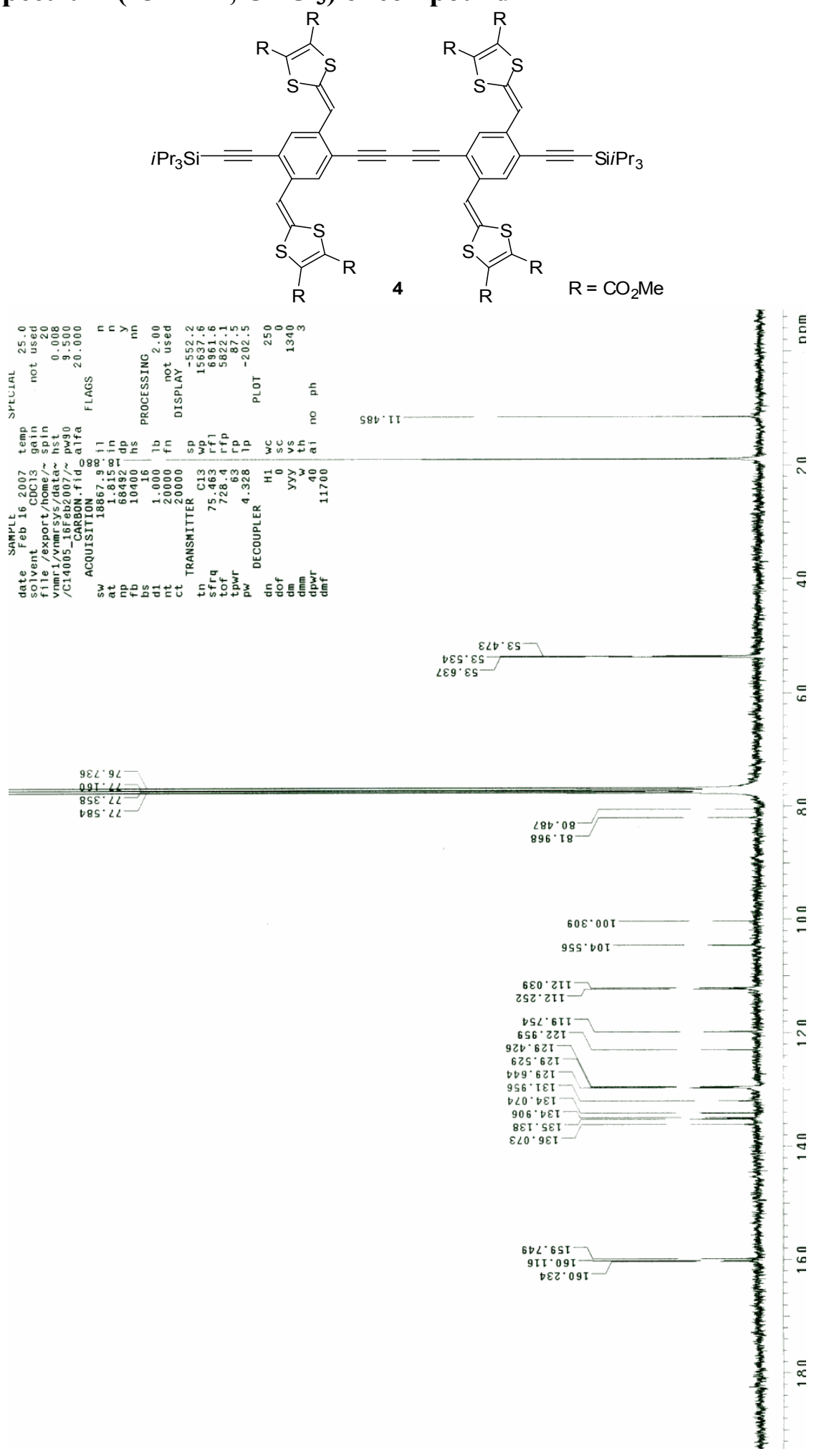
${ }^{1} \mathrm{H}$ NMR Spectrum (300 MHz, $\mathrm{CDCl}_{3}$ ) of compound 13
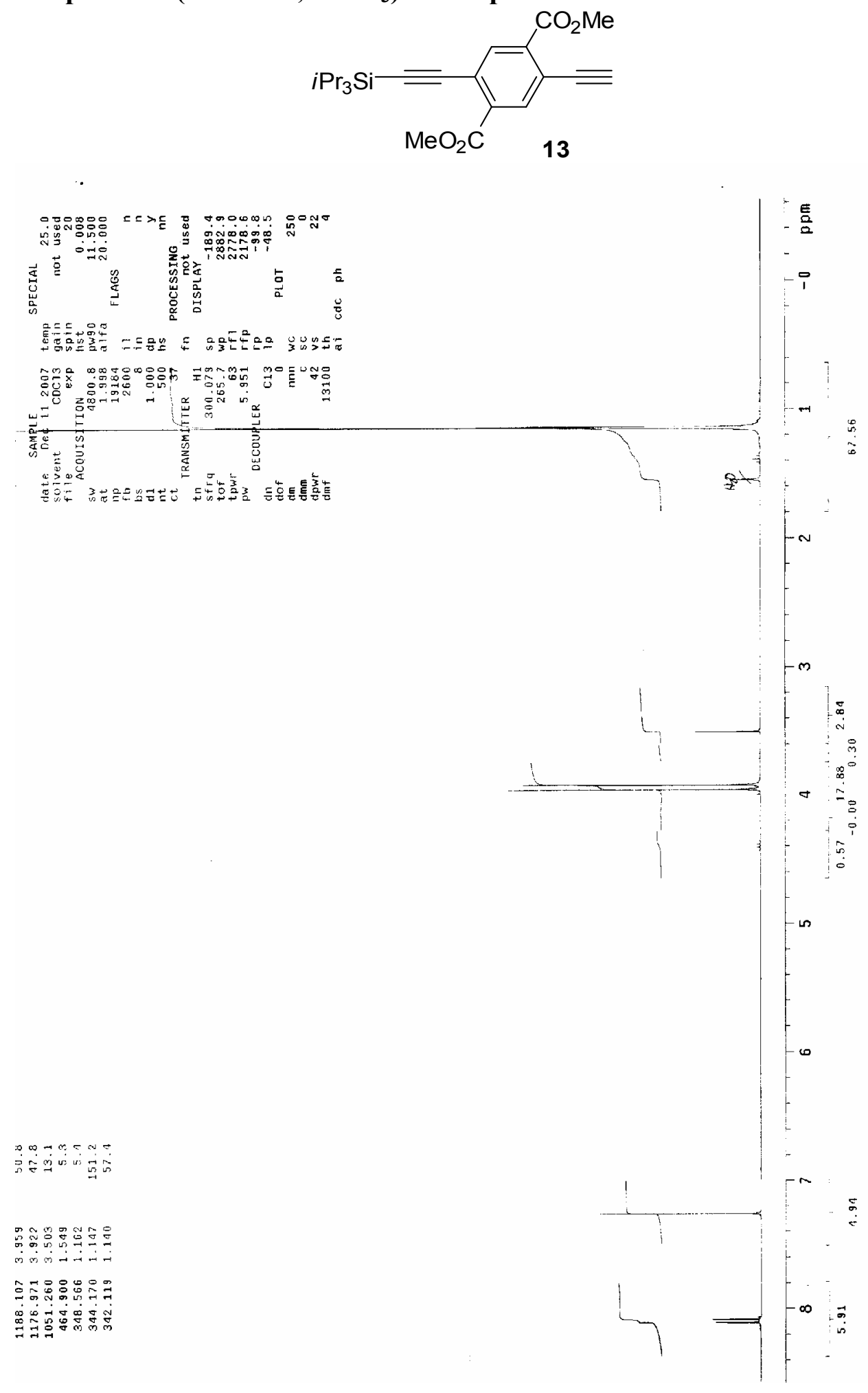


\section{${ }^{13} \mathrm{C}$ NMR Spectrum (75 MHz, $\mathrm{CDCl}_{3}$ ) of compound 13}
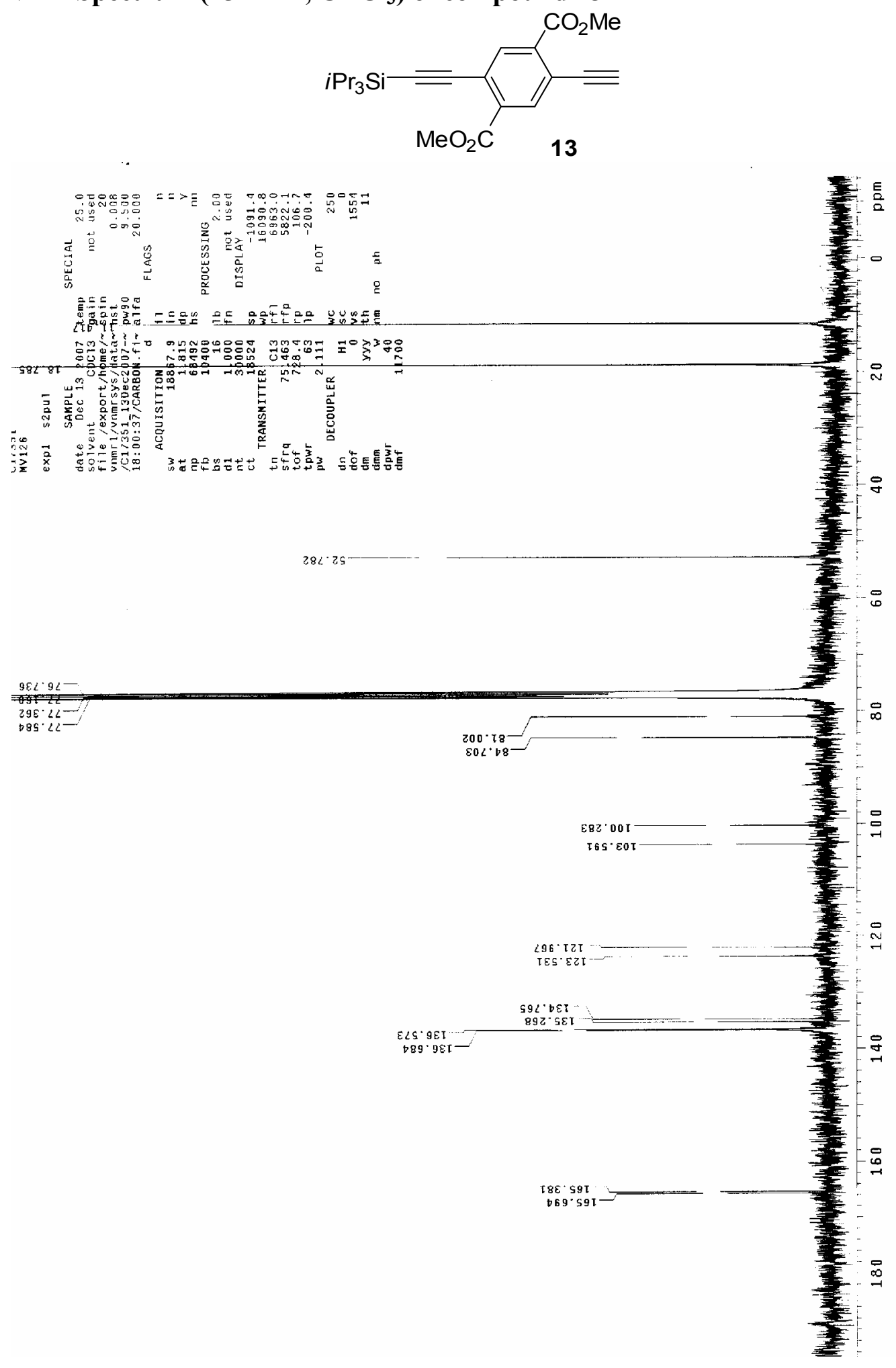
${ }^{1} \mathrm{H}$ NMR Spectrum (300 MHz, $\mathrm{CDCl}_{3}$ ) of compound 14
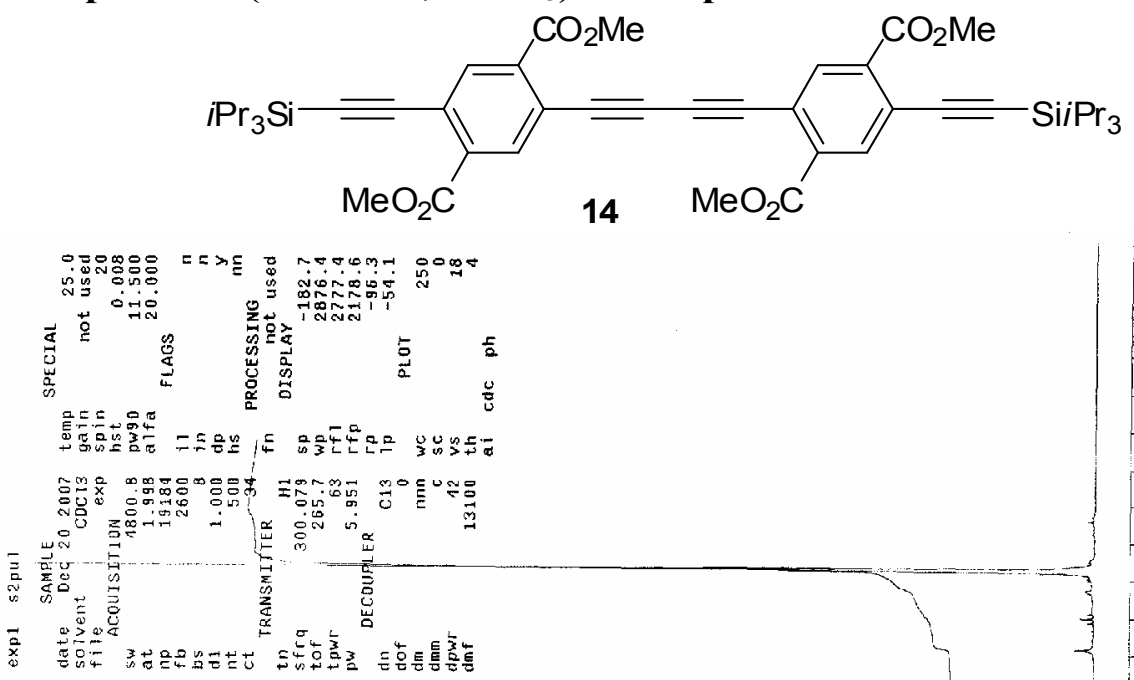

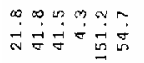

踢两品品

的的的

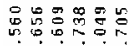

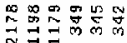

tenconos

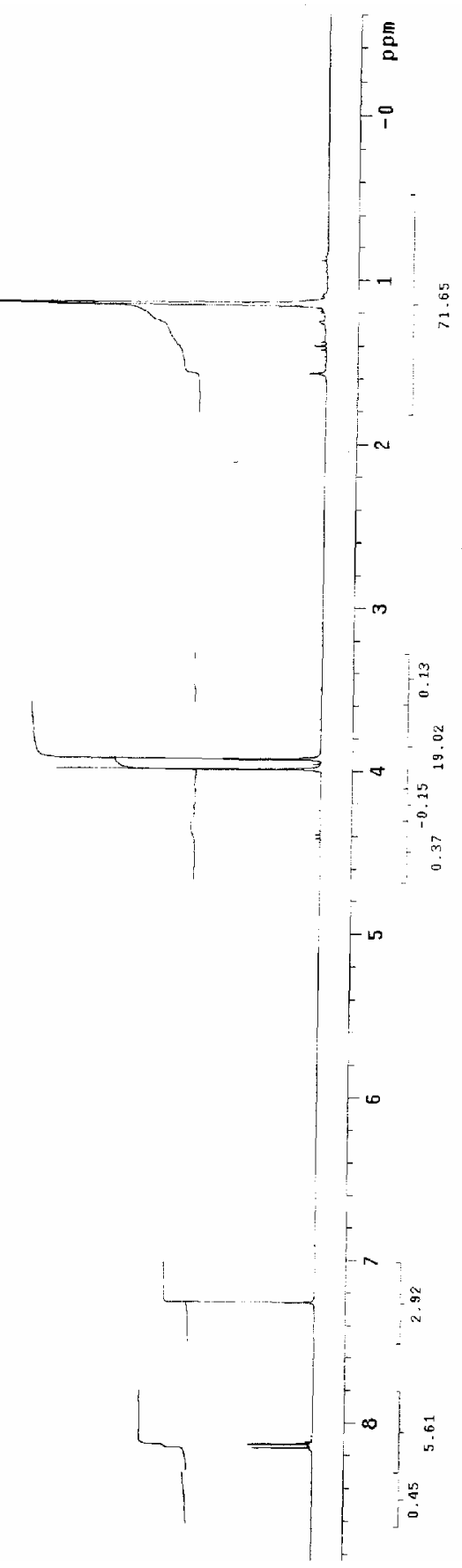


${ }^{13} \mathrm{C}$ NMR Spectrum (75 MHz, $\mathrm{CDCl}_{3}$ ) of compound 14
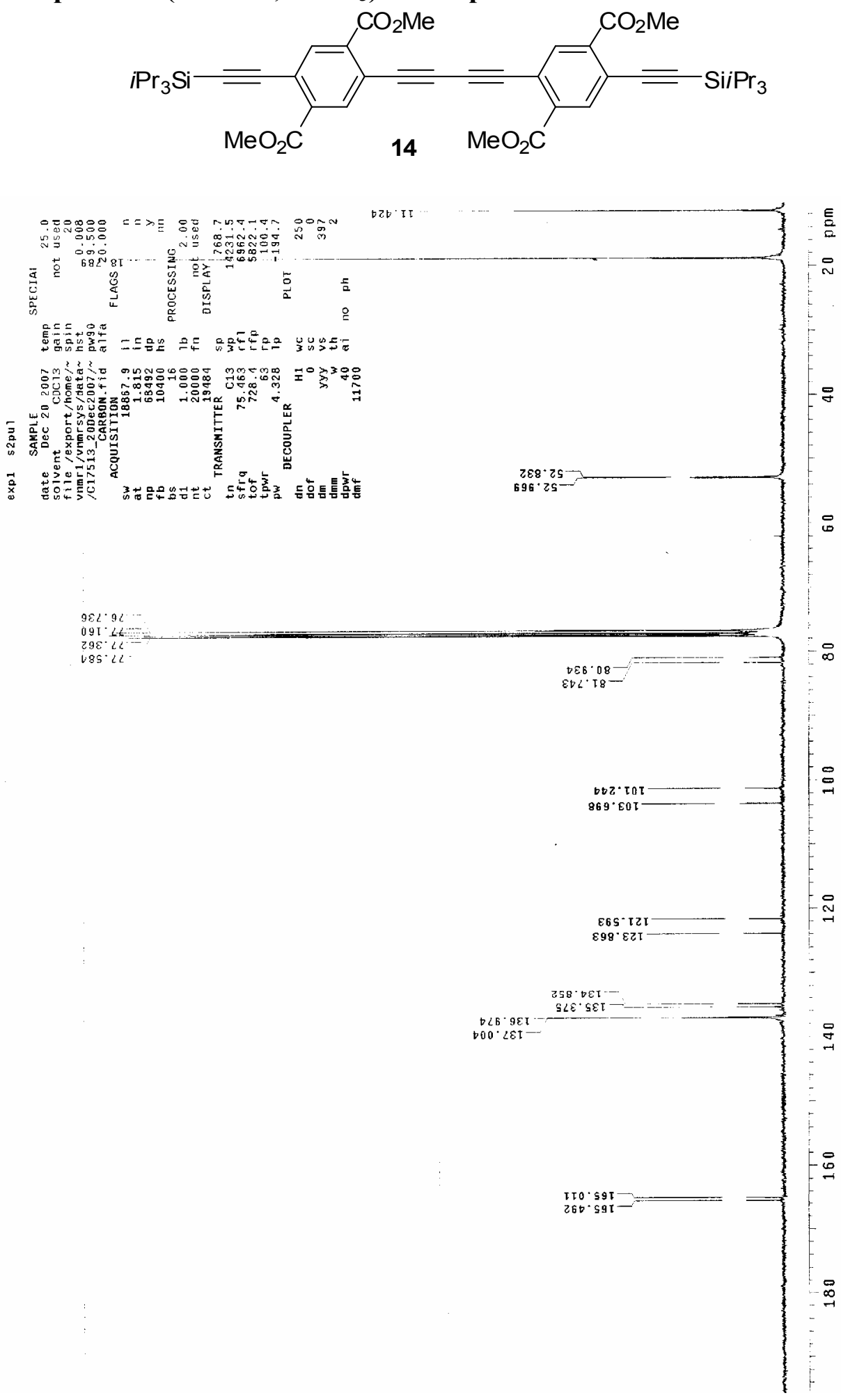

$298 \cdot 2 i$

$14 \quad \mathrm{MeO}_{2} \mathrm{C}$

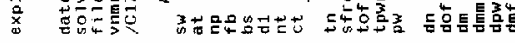

. II

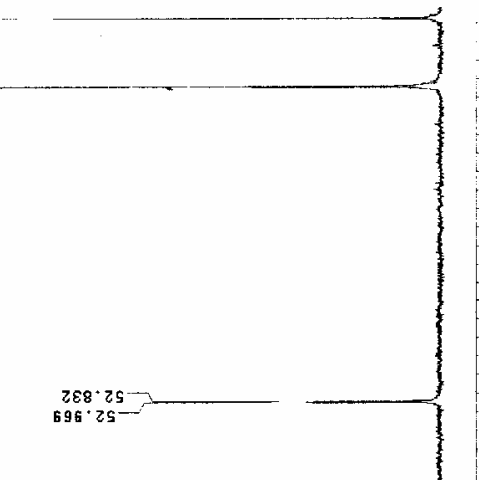

E⿳亠二口⿱口小

กิ 
${ }^{1} \mathrm{H}$ NMR Spectrum (300 MHz, $\mathrm{CDCl}_{3}$ ) of compound 15
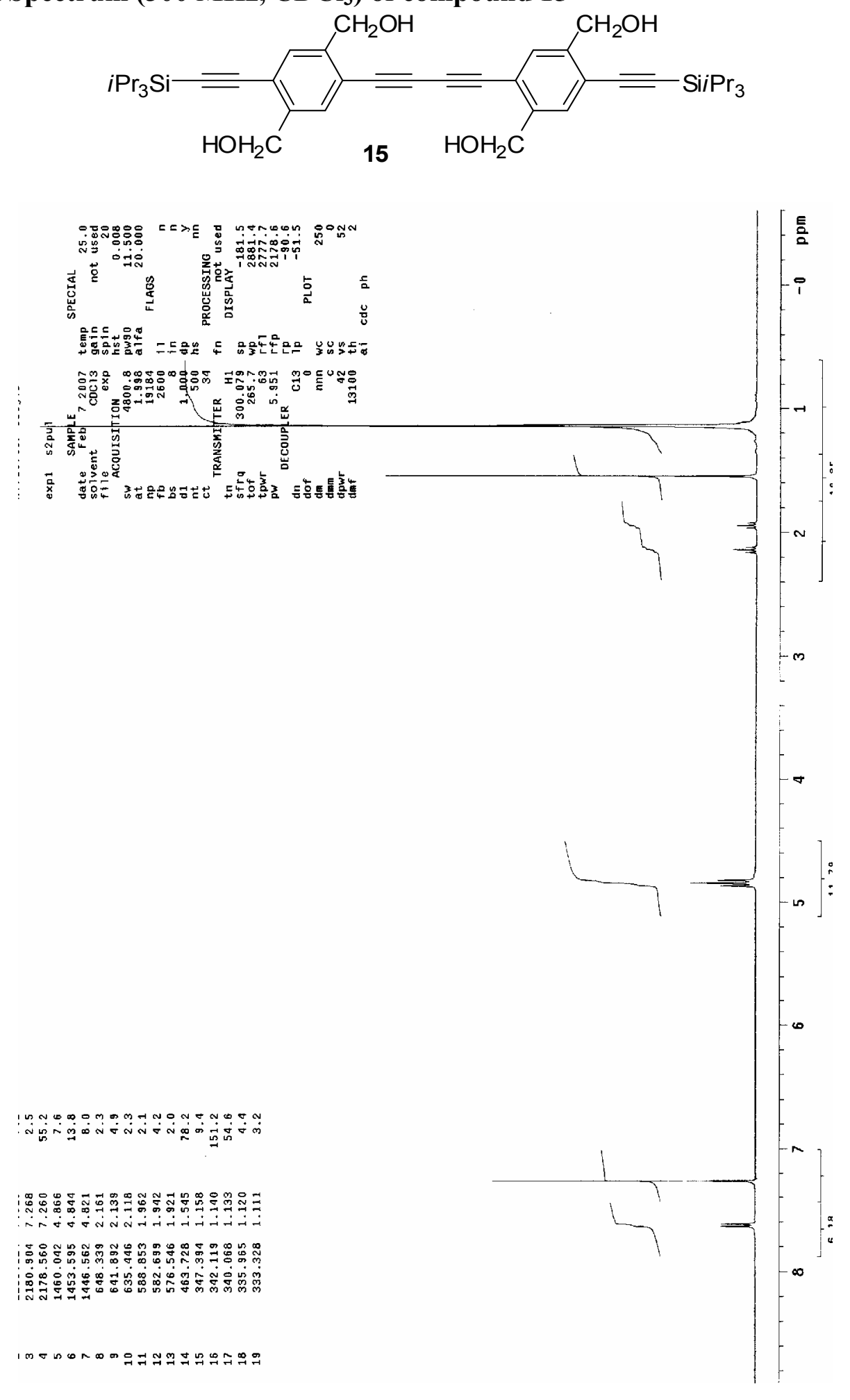
${ }^{13} \mathrm{C}$ NMR Spectrum (75 $\mathrm{MHz}, \mathrm{CDCl}_{3}$ ) of compound 15
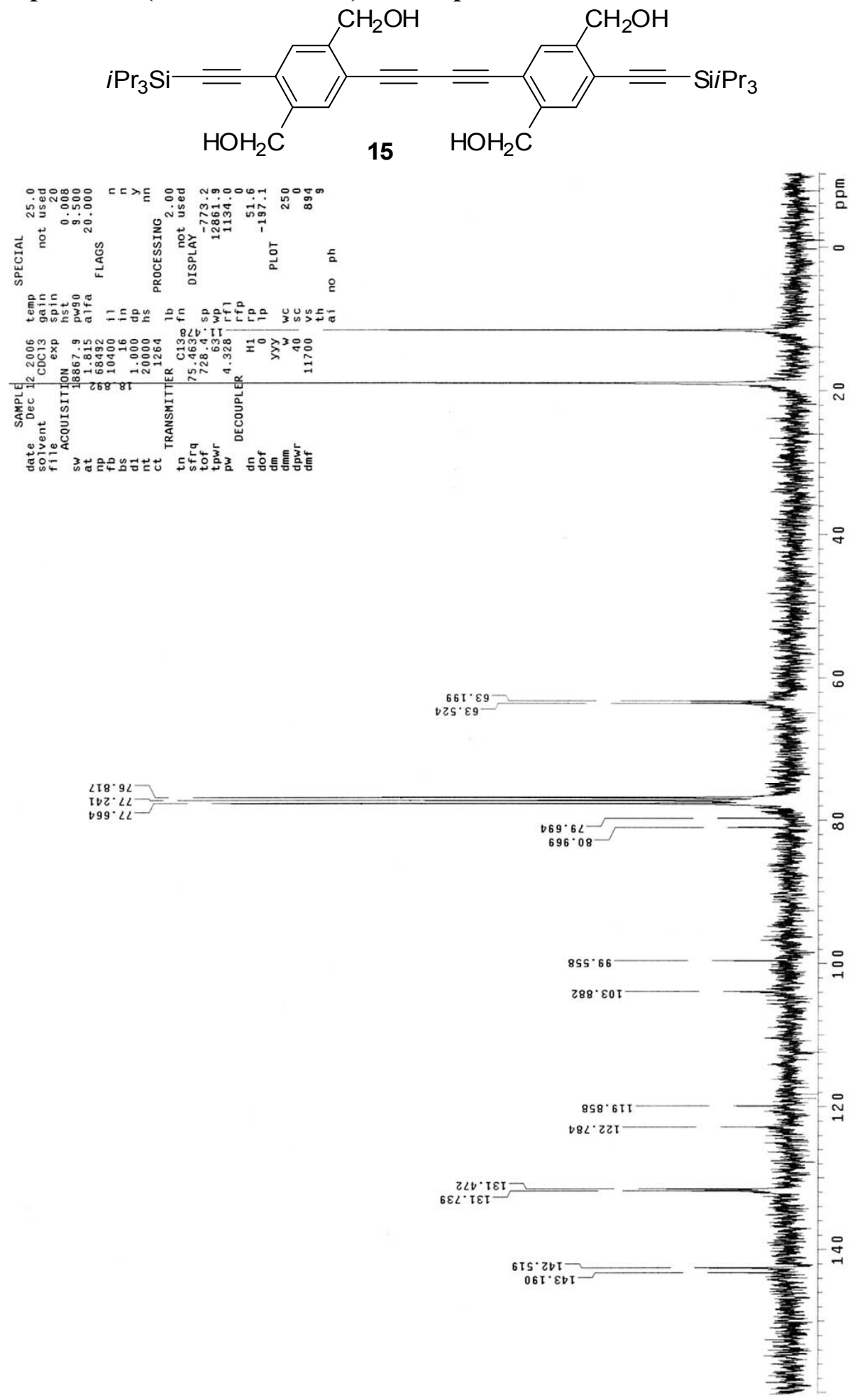
${ }^{1} \mathrm{H}$ NMR Spectrum (300 MHz, $\mathrm{CDCl}_{3}$ ) of compound 5
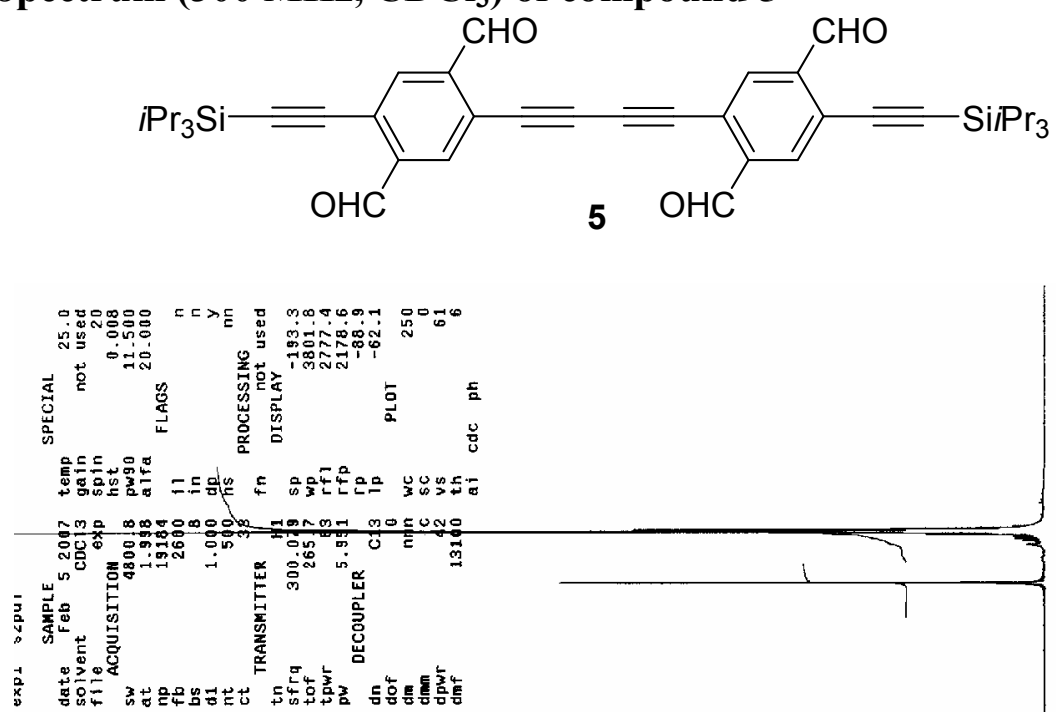

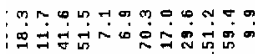

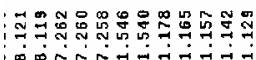

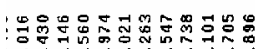

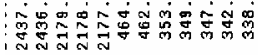

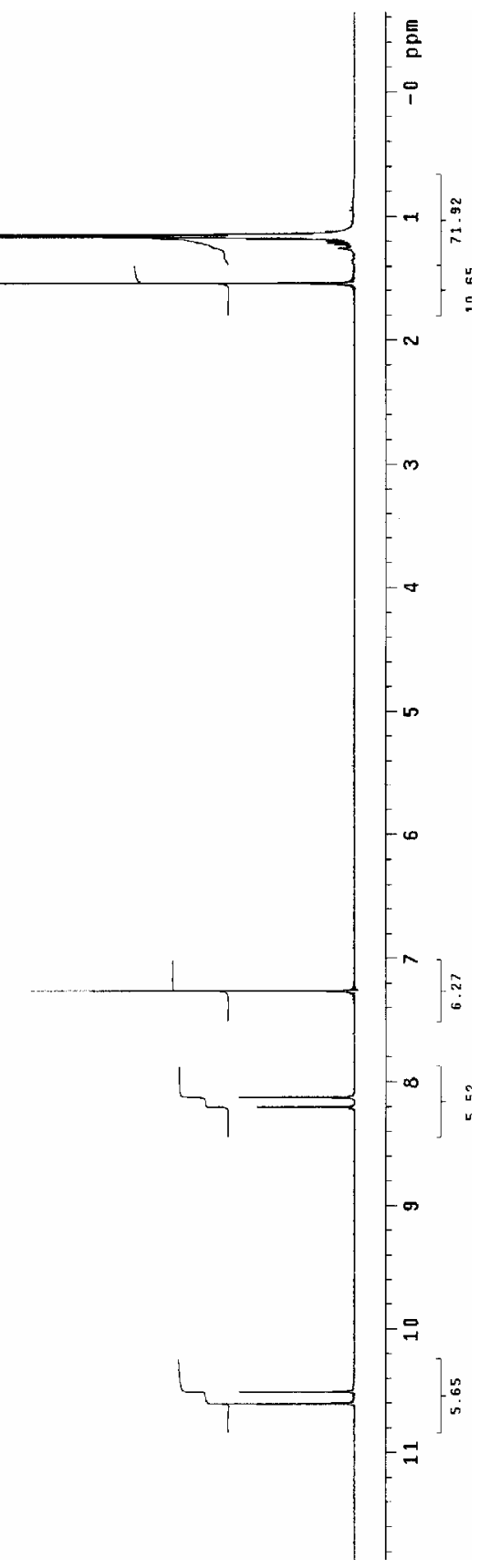


${ }^{13} \mathrm{C}$ NMR Spectrum (75 MHz, $\mathrm{CDCl}_{3}$ ) of compound 5<smiles>Cc1cc(C#C[Sn]C(C)C)c(C=O)cc1C#CC#Cc1cc(C=O)c(C=O)cc1C=O</smiles>
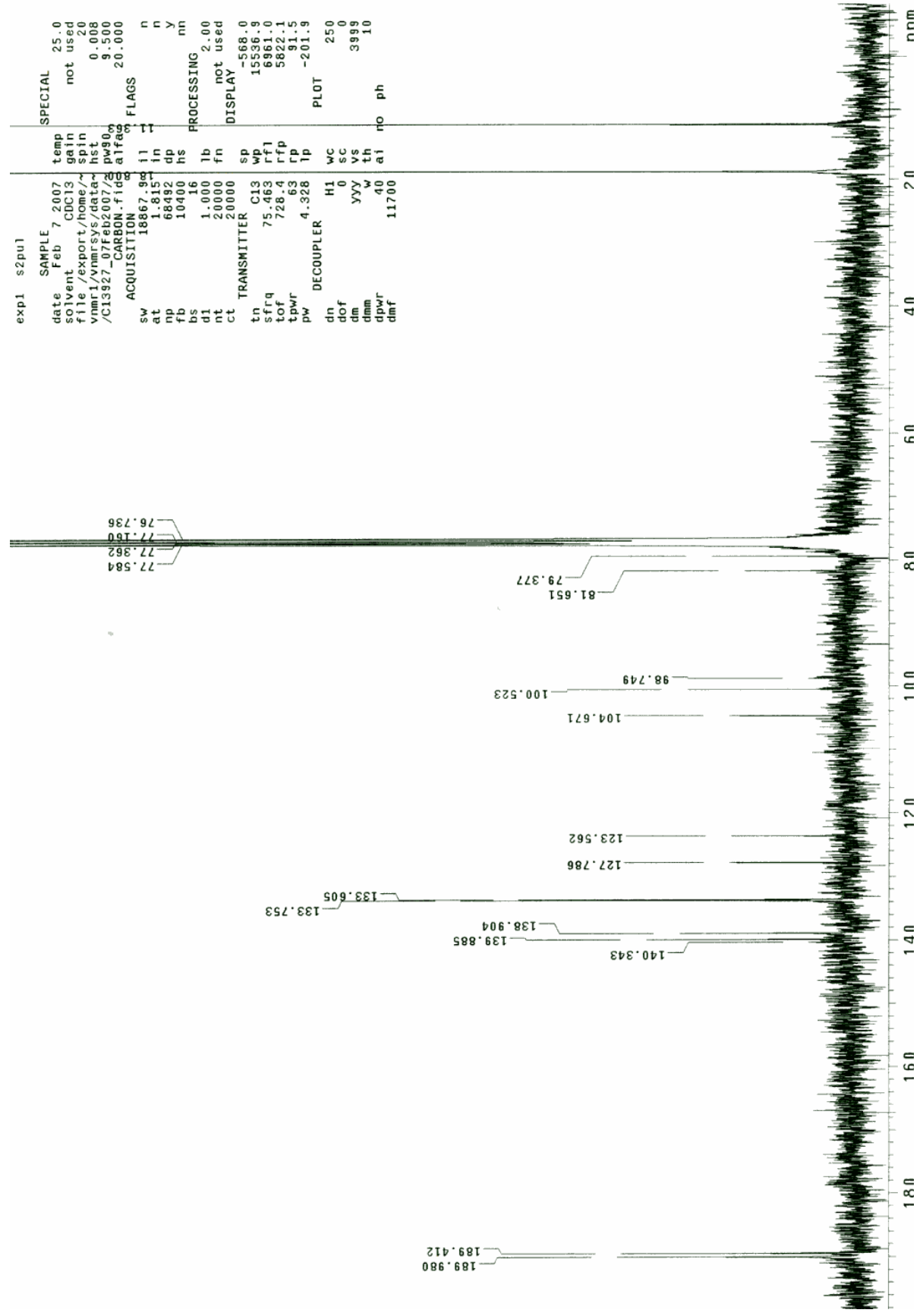
${ }^{1} \mathrm{H}$ NMR Spectrum (300 MHz, $\mathrm{CDCl}_{3}$ ) of compound 16
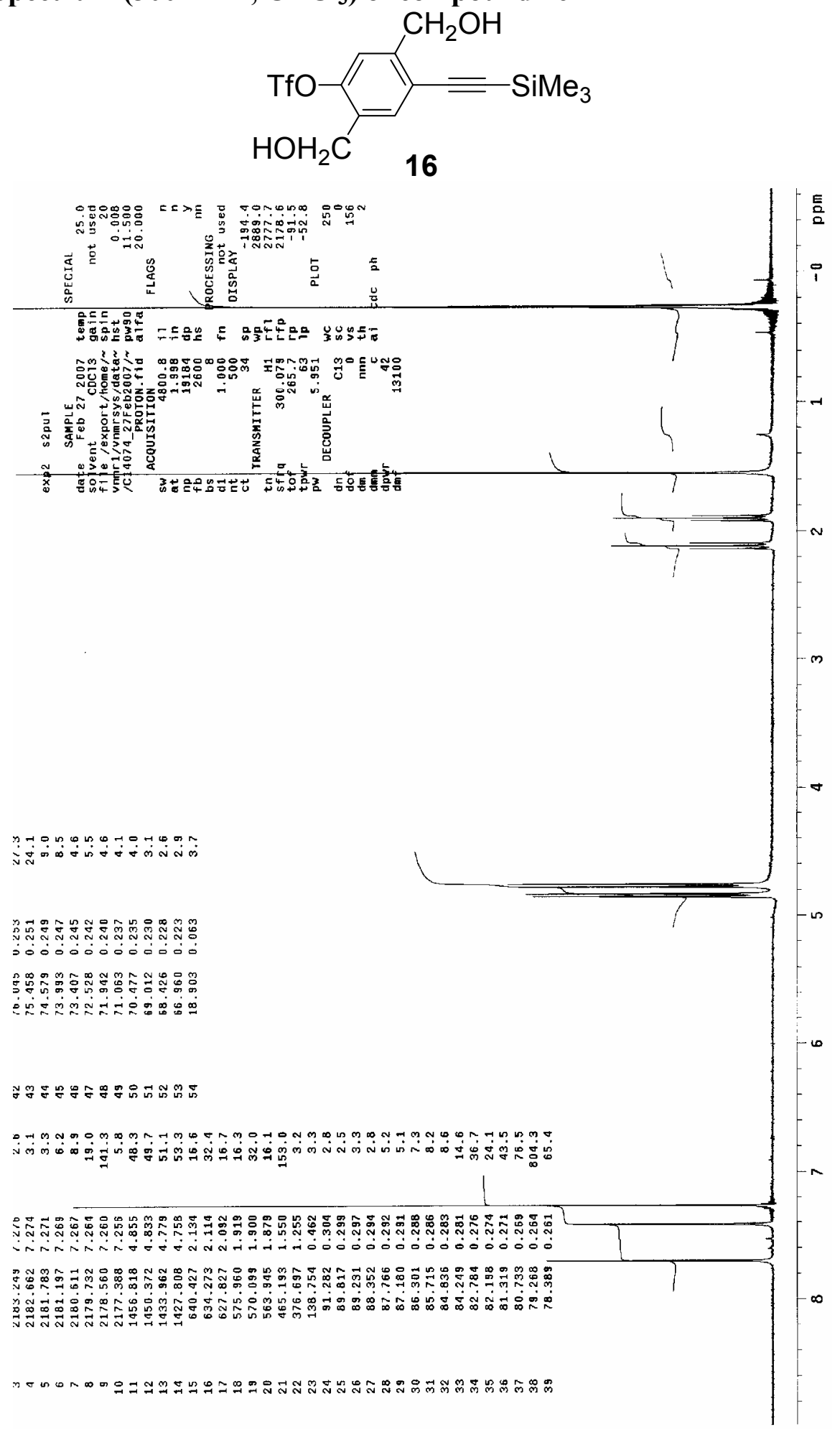
${ }^{13} \mathrm{C}$ NMR Spectrum (75 MHz, $\mathrm{CDCl}_{3}$ ) of compound 16

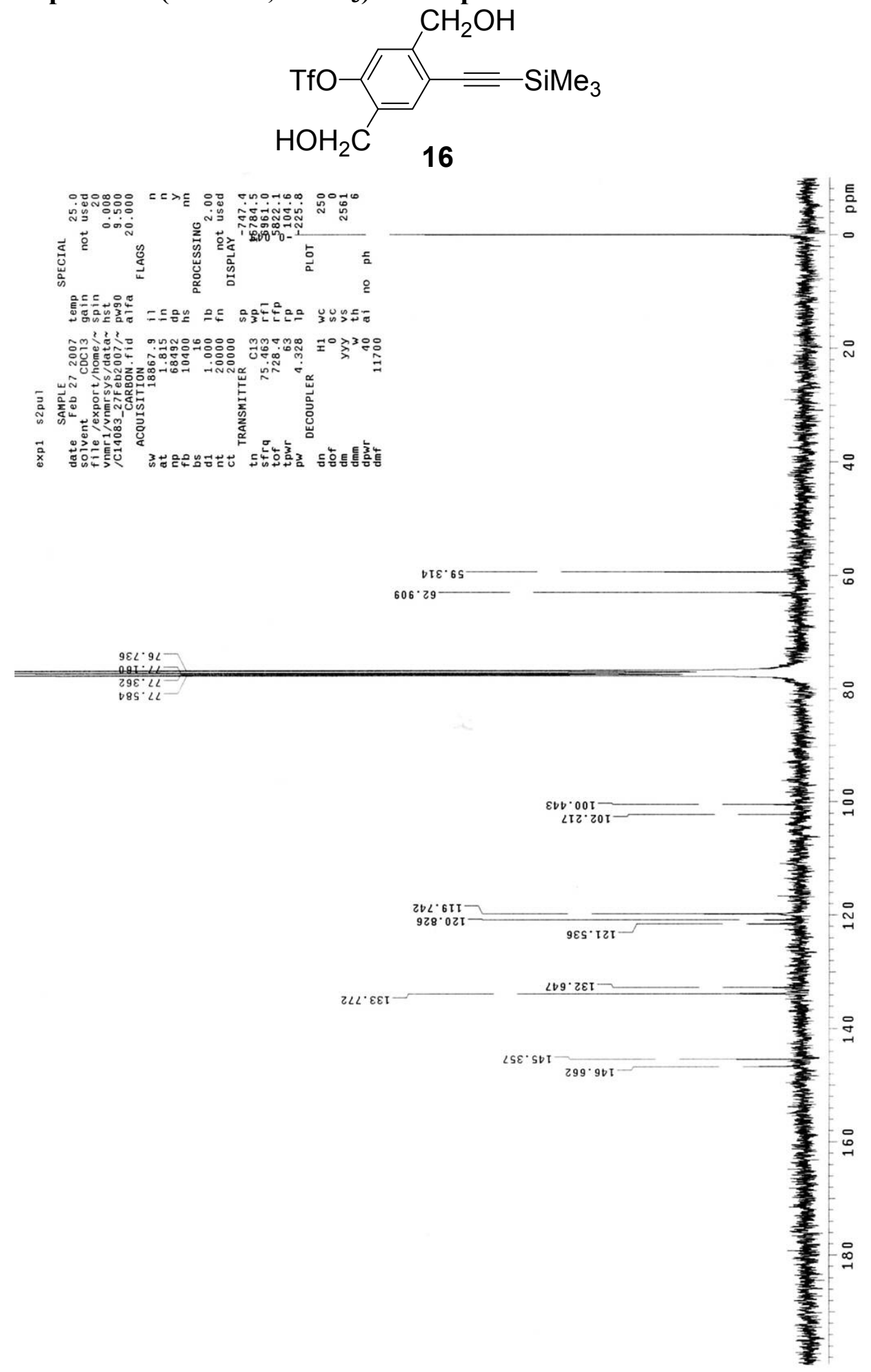


${ }^{1} \mathrm{H}$ NMR Spectrum (300 MHz, $\mathrm{CDCl}_{3}$ ) of compound 17
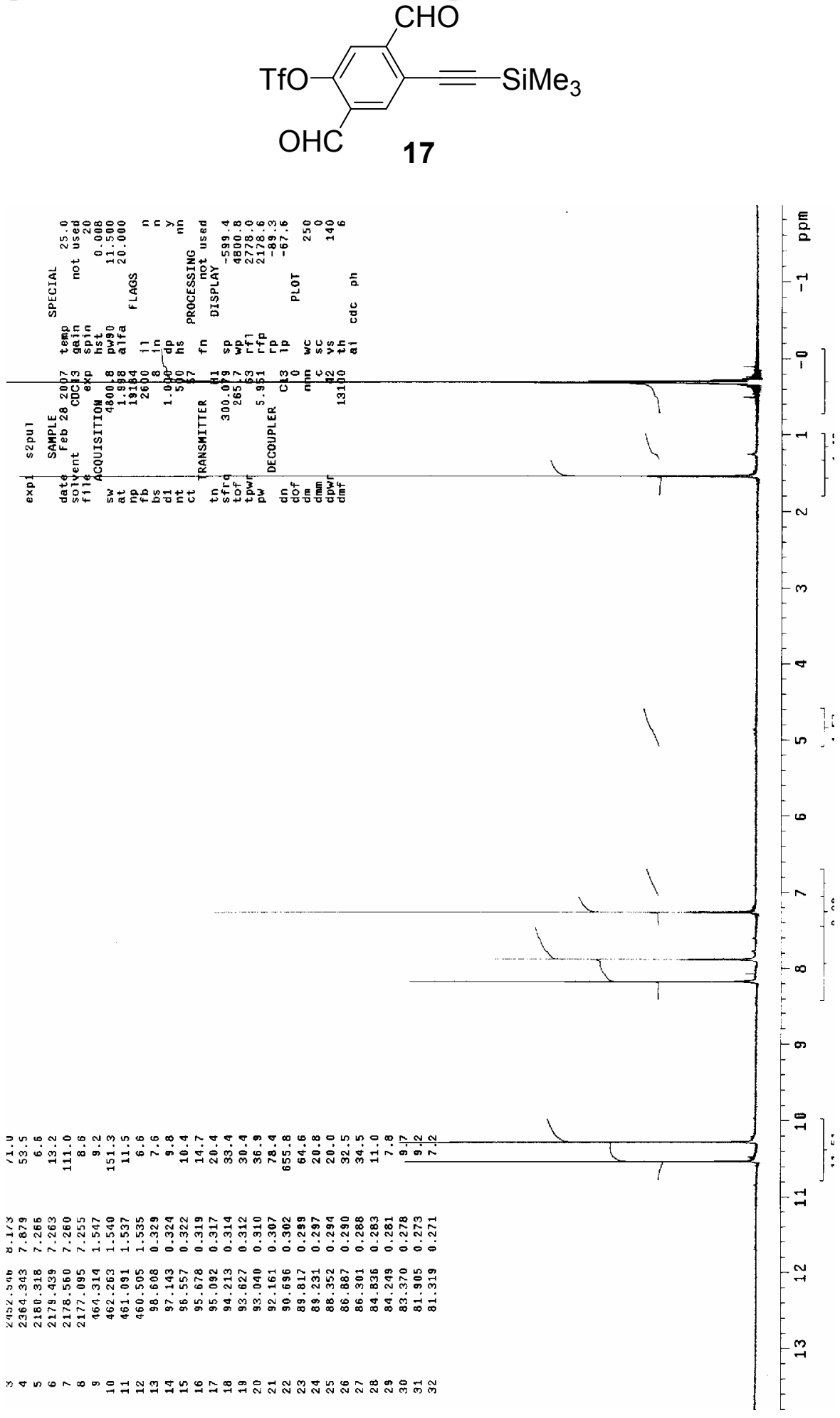


\section{${ }^{13} \mathrm{C}$ NMR Spectrum (75 MHz, $\mathrm{CDCl}_{3}$ ) of compound 17}
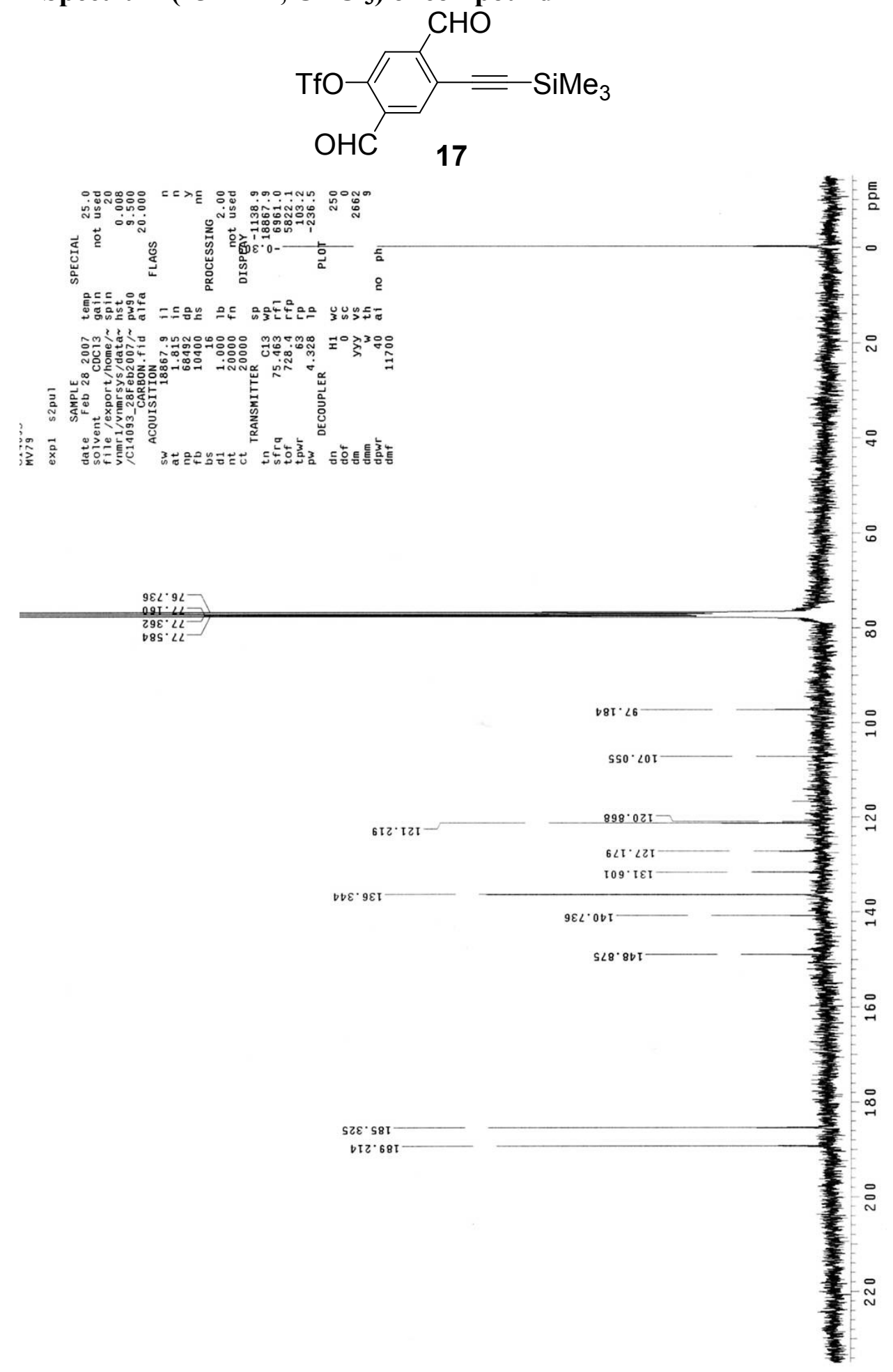
${ }^{1} \mathrm{H}$ NMR Spectrum (300 MHz, $\mathrm{CDCl}_{3}$ ) of compound 18
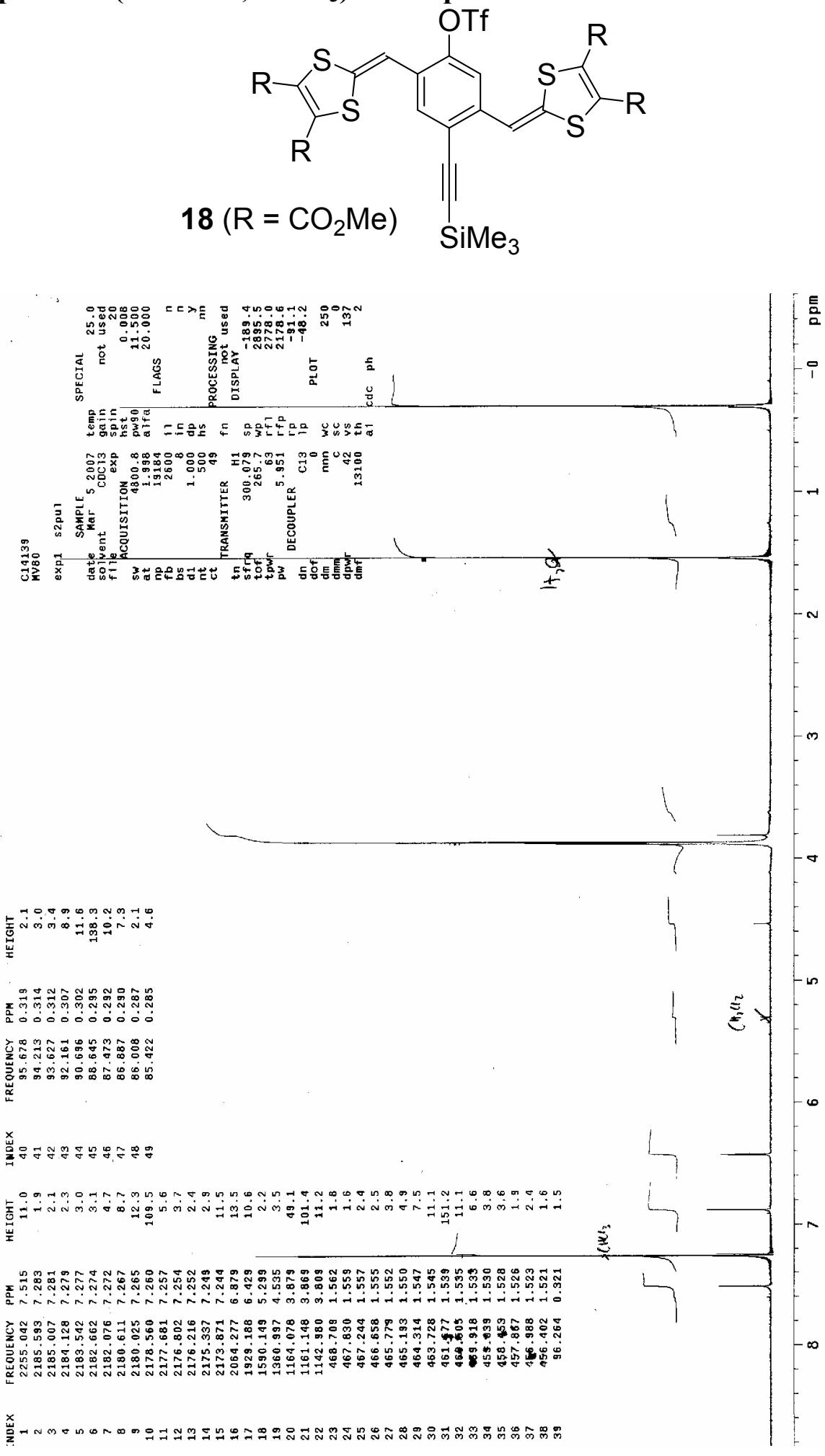


\section{Atom Coordinates and Absolute Energies (Calculated using Gaussian 03)}

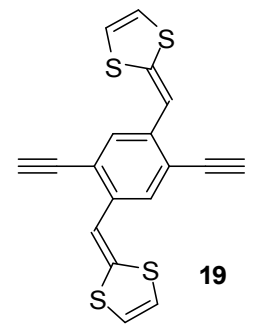

\section{B3LYP/6-31G(d)}

$\mathrm{HF}=-2284.4886967$ Hartrees

\begin{tabular}{|c|c|c|c|c|c|}
\hline \multirow{2}{*}{$\begin{array}{l}\text { Center } \\
\text { Number }\end{array}$} & \multirow{2}{*}{$\begin{array}{l}\text { Atomic } \\
\text { Number }\end{array}$} & \multirow{2}{*}{$\begin{array}{l}\text { Atomic } \\
\text { Type }\end{array}$} & \multicolumn{3}{|c|}{ Coordinates (Angstroms) } \\
\hline & & & $\mathrm{x}$ & $\mathrm{Y}$ & Z \\
\hline 1 & 6 & 0 & 1.373232 & 0.441661 & 0.086630 \\
\hline 2 & 6 & 0 & 1.022184 & -0.913713 & 0.186921 \\
\hline 3 & 6 & 0 & -0.299399 & -1.366237 & 0.099497 \\
\hline 4 & 6 & 0 & -1.373232 & -0.441659 & -0.086637 \\
\hline 5 & 6 & 0 & -1.022184 & 0.913716 & -0.186929 \\
\hline 6 & 6 & 0 & 0.299399 & 1.366239 & -0.099505 \\
\hline 7 & 1 & 0 & 1.783500 & -1.660347 & 0.374818 \\
\hline 8 & 1 & 0 & -1.783499 & 1.660349 & -0.374828 \\
\hline 9 & 6 & 0 & -0.539093 & -2.769883 & 0.219958 \\
\hline 10 & 6 & 0 & -0.719318 & -3.962932 & 0.322015 \\
\hline 11 & 1 & 0 & -0.874461 & -5.013504 & 0.417690 \\
\hline 12 & 6 & 0 & 0.539093 & 2.769885 & -0.219968 \\
\hline 13 & 6 & 0 & 0.719318 & 3.962934 & -0.322026 \\
\hline 14 & 1 & 0 & 0.874461 & 5.013506 & -0.417702 \\
\hline 15 & 6 & 0 & -2.732333 & -0.947985 & -0.176533 \\
\hline 16 & 6 & 0 & 2.732334 & 0.947987 & 0.176525 \\
\hline 17 & 6 & 0 & 3.923010 & 0.302408 & 0.095484 \\
\hline 18 & 6 & 0 & 5.960521 & -1.324172 & -0.276054 \\
\hline 19 & 6 & 0 & 6.519388 & -0.129544 & -0.052889 \\
\hline 20 & 1 & 0 & 6.504455 & -2.238195 & -0.483748 \\
\hline 21 & 1 & 0 & 7.585295 & 0.066431 & -0.054007 \\
\hline 22 & 6 & 0 & -3.923010 & -0.302408 & -0.095486 \\
\hline 23 & 6 & 0 & -6.519388 & 0.129540 & 0.052903 \\
\hline 24 & 6 & 0 & -5.960521 & 1.324167 & 0.276073 \\
\hline 25 & 1 & 0 & -7.585295 & -0.066437 & 0.054025 \\
\hline 26 & 1 & 0 & -6.504456 & 2.238188 & 0.483778 \\
\hline 27 & 1 & 0 & 2.798063 & 2.025614 & 0.304623 \\
\hline 28 & 1 & 0 & -2.798062 & -2.025612 & -0.304636 \\
\hline 29 & 16 & 0 & 4.203222 & -1.430502 & -0.220704 \\
\hline 30 & 16 & 0 & 5.447955 & 1.220400 & 0.280655 \\
\hline 31 & 16 & 0 & -5.447954 & -1.220400 & -0.280657 \\
\hline 32 & 16 & 0 & -4.203224 & 1.430500 & 0.220715 \\
\hline
\end{tabular}




\section{Atom Coordinates and Absolute Energies (Calculated using Gaussian 03)}

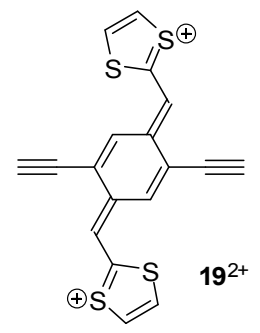

B3LYP/6-31G(d)

$\mathrm{HF}=-2283.9371605$ Hartrees

\begin{tabular}{|c|c|c|c|c|c|}
\hline \multirow{2}{*}{$\begin{array}{l}\text { Center } \\
\text { Number }\end{array}$} & \multirow{2}{*}{$\begin{array}{l}\text { Atomic } \\
\text { Number }\end{array}$} & \multirow{2}{*}{$\begin{array}{l}\text { Atomic } \\
\text { Type }\end{array}$} & \multicolumn{3}{|c|}{ Coordinates (Angstroms) } \\
\hline & & & $\mathrm{X}$ & $\mathrm{Y}$ & Z \\
\hline 1 & 6 & 0 & 1.386560 & 0.412024 & 0.000182 \\
\hline 2 & 6 & 0 & 1.017060 & -0.967610 & 0.000718 \\
\hline 3 & 6 & 0 & -0.290858 & -1.394745 & 0.000518 \\
\hline 4 & 6 & 0 & -1.386556 & -0.412013 & -0.000197 \\
\hline 5 & 6 & 0 & -1.017056 & 0.967621 & -0.000733 \\
\hline 6 & 6 & 0 & 0.290862 & 1.394756 & -0.000534 \\
\hline 7 & 1 & 0 & 1.779121 & -1.735306 & 0.001558 \\
\hline 8 & 1 & 0 & -1.779116 & 1.735317 & -0.001573 \\
\hline 9 & 6 & 0 & -0.564444 & -2.787843 & 0.001212 \\
\hline 10 & 6 & 0 & -0.773297 & -3.980883 & 0.001344 \\
\hline 11 & 1 & 0 & -0.945297 & -5.037343 & 0.001674 \\
\hline 12 & 6 & 0 & 0.564447 & 2.787854 & -0.001228 \\
\hline 13 & 6 & 0 & 0.773299 & 3.980894 & -0.001361 \\
\hline 14 & 1 & 0 & 0.945299 & 5.037355 & -0.001691 \\
\hline 15 & 6 & 0 & -2.692067 & -0.902065 & -0.000346 \\
\hline 16 & 6 & 0 & 2.692072 & 0.902072 & 0.000331 \\
\hline 17 & 6 & 0 & 3.949951 & 0.247058 & 0.000172 \\
\hline 18 & 6 & 0 & 6.012885 & -1.309852 & -0.000818 \\
\hline 19 & 6 & 0 & 6.518348 & -0.053934 & 0.000382 \\
\hline 20 & 1 & 0 & 6.595934 & -2.224843 & -0.001519 \\
\hline 21 & 1 & 0 & 7.574306 & 0.195825 & 0.000824 \\
\hline 22 & 6 & 0 & -3.949950 & -0.247057 & -0.000188 \\
\hline 23 & 6 & 0 & -6.518350 & 0.053916 & -0.000298 \\
\hline 24 & 6 & 0 & -6.012896 & 1.309838 & 0.000901 \\
\hline 25 & 1 & 0 & -7.574306 & -0.195850 & -0.000687 \\
\hline 26 & 1 & 0 & -6.595952 & 2.224824 & 0.001655 \\
\hline 27 & 1 & 0 & 2.770176 & 1.986921 & 0.000485 \\
\hline 28 & 1 & 0 & -2.770166 & -1.986914 & -0.000499 \\
\hline 29 & 16 & 0 & 4.285464 & -1.448138 & -0.001422 \\
\hline 30 & 16 & 0 & 5.371969 & 1.237648 & 0.001299 \\
\hline 31 & 16 & 0 & -5.371961 & -1.237657 & -0.001316 \\
\hline 32 & 16 & 0 & -4.285476 & 1.448136 & 0.001404 \\
\hline
\end{tabular}




\section{Atom Coordinates and Absolute Energies (Calculated using Gaussian 03)}

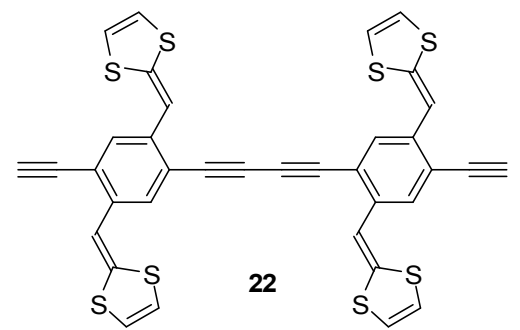

B3LYP/6-31G(d)

$\mathrm{HF}=-4567.8117499$ Hartrees

\begin{tabular}{|c|c|c|c|c|c|}
\hline \multirow{2}{*}{$\begin{array}{l}\text { Center } \\
\text { Number }\end{array}$} & \multirow{2}{*}{$\begin{array}{l}\text { Atomic } \\
\text { Number }\end{array}$} & \multirow{2}{*}{$\begin{array}{l}\text { Atomic } \\
\text { Type }\end{array}$} & \multicolumn{3}{|c|}{ Coordinates (Angstroms) } \\
\hline & & & $\mathrm{X}$ & $\mathrm{Y}$ & Z \\
\hline 1 & 6 & 0 & 8.586213 & -1.751803 & -0.006459 \\
\hline 2 & 6 & 0 & 7.406172 & -1.479735 & -0.014447 \\
\hline 3 & 6 & 0 & 6.008025 & -1.186715 & -0.019298 \\
\hline 4 & 6 & 0 & 5.522602 & 0.123767 & -0.325643 \\
\hline 5 & 6 & 0 & 4.131807 & 0.298531 & -0.293401 \\
\hline 6 & 6 & 0 & 3.238841 & -0.734776 & 0.029993 \\
\hline 7 & 6 & 0 & 3.726738 & -2.046543 & 0.334854 \\
\hline 8 & 6 & 0 & 5.118963 & -2.218722 & 0.302105 \\
\hline 9 & 6 & 0 & 1.850113 & -0.441892 & 0.031207 \\
\hline 10 & 6 & 0 & 0.660378 & -0.157333 & 0.027628 \\
\hline 11 & 6 & 0 & 2.775465 & -3.090274 & 0.671420 \\
\hline 12 & 6 & 0 & 2.933007 & -4.435304 & 0.765885 \\
\hline 13 & 6 & 0 & 6.477576 & 1.166747 & -0.659609 \\
\hline 14 & 6 & 0 & 6.321456 & 2.510688 & -0.764711 \\
\hline 15 & 16 & 0 & 7.714600 & 3.533865 & -1.227732 \\
\hline 16 & 6 & 0 & 6.880441 & 5.069531 & -1.063958 \\
\hline 17 & 6 & 0 & 5.587679 & 5.039310 & -0.721516 \\
\hline 18 & 16 & 0 & 4.845503 & 3.463647 & -0.461039 \\
\hline 19 & 16 & 0 & 1.547573 & -5.462131 & 1.242170 \\
\hline 20 & 6 & 0 & 2.378983 & -6.996158 & 1.052197 \\
\hline 21 & 6 & 0 & 3.666581 & -6.963200 & 0.690903 \\
\hline 22 & 16 & 0 & 4.404891 & -5.385254 & 0.433745 \\
\hline 23 & 6 & 0 & -0.660382 & 0.157343 & 0.027629 \\
\hline 24 & 6 & 0 & -1.850116 & 0.441901 & 0.031208 \\
\hline 25 & 6 & 0 & -3.238844 & 0.734781 & 0.029995 \\
\hline 26 & 6 & 0 & -3.726744 & 2.046545 & 0.334857 \\
\hline 27 & 6 & 0 & -5.118970 & 2.218720 & 0.302109 \\
\hline 28 & 6 & 0 & -6.008028 & 1.186711 & -0.019293 \\
\hline 29 & 6 & 0 & -5.522602 & -0.123769 & -0.325640 \\
\hline 30 & 6 & 0 & -4.131806 & -0.298529 & -0.293400 \\
\hline 31 & 6 & 0 & -7.406175 & 1.479726 & -0.014442 \\
\hline 32 & 6 & 0 & -6.477573 & -1.166751 & -0.659607 \\
\hline 33 & 6 & 0 & -2.775472 & 3.090277 & 0.671422 \\
\hline
\end{tabular}




$\begin{array}{rrrrrr}34 & 6 & 0 & -6.321452 & -2.510692 & -0.764713 \\ 35 & 6 & 0 & -2.933012 & 4.435308 & 0.765888 \\ 36 & 16 & 0 & -7.714597 & -3.533868 & -1.227734 \\ 37 & 6 & 0 & -6.880438 & -5.069534 & -1.063962 \\ 38 & 6 & 0 & -5.587675 & -5.039315 & -0.721521 \\ 39 & 16 & 0 & -4.845499 & -3.463651 & -0.461043 \\ 40 & 16 & 0 & -1.547574 & 5.462132 & 1.242167 \\ 41 & 6 & 0 & -2.378976 & 6.996161 & 1.052186 \\ 42 & 6 & 0 & -3.666575 & 6.963207 & 0.690894 \\ 43 & 16 & 0 & -4.404892 & 5.385263 & 0.433745 \\ 44 & 6 & 0 & -8.586218 & 1.751790 & -0.006454 \\ 45 & 1 & 0 & 9.624708 & -1.993298 & 0.006794 \\ 46 & 1 & 0 & 3.695867 & 1.253568 & -0.557432 \\ 47 & 1 & 0 & 5.555341 & -3.173502 & 0.566911 \\ 48 & 1 & 0 & 1.766231 & -2.729768 & 0.855603 \\ 49 & 1 & 0 & 7.488240 & 0.804487 & -0.830170 \\ 50 & 1 & 0 & 7.450304 & 5.971129 & -1.256031 \\ 51 & 1 & 0 & 4.954907 & 5.910050 & -0.595872 \\ 52 & 1 & 0 & 1.812183 & -7.899545 & 1.244998 \\ 53 & 1 & 0 & 4.297061 & -7.832972 & 0.548056 \\ 54 & 1 & 0 & -5.555351 & 3.173498 & 0.566917 \\ 55 & 1 & 0 & -3.695863 & -1.253564 & -0.557432 \\ 56 & 1 & 0 & -7.488238 & -0.804492 & -0.830167 \\ 57 & 1 & 0 & -1.766238 & 2.729772 & 0.855604 \\ 58 & 1 & 0 & -7.450301 & -5.971133 & -1.256036 \\ 59 & 1 & 0 & -4.954904 & -5.910055 & -0.595878 \\ 60 & 1 & 0 & -1.812171 & 7.899547 & 1.244981 \\ 61 & 1 & 0 & -4.297051 & 7.832982 & 0.548042 \\ 62 & 1 & 0 & -9.624714 & 1.993282 & 0.006800 \\ --------------1 & \end{array}$


Atom Coordinates and Absolute Energies (Calculated using Gaussian 03)<smiles>c1ccc(CC2CC2C2CCCC2)cc1</smiles>

B3LYP/6-311++G(2d,p)

$z=0: \mathrm{HF}=-232.3174859$

$z=1: \mathrm{HF}=-231.9763514$ (same geometry used as obtained for neutral structure)

\begin{tabular}{|c|c|c|c|c|c|}
\hline \multirow{2}{*}{$\begin{array}{l}\text { Center } \\
\text { Number }\end{array}$} & \multirow{2}{*}{$\begin{array}{l}\text { Atomic } \\
\text { Number }\end{array}$} & \multirow{2}{*}{$\begin{array}{l}\text { Atomic } \\
\text { Type }\end{array}$} & \multicolumn{3}{|c|}{ Coordinates (Angstroms) } \\
\hline & & & $\mathrm{x}$ & $\mathrm{Y}$ & Z \\
\hline 1 & 6 & 0 & -1.388424 & 0.100487 & -0.000001 \\
\hline 2 & 6 & 0 & -0.607050 & 1.252428 & -0.000009 \\
\hline 3 & 6 & 0 & 0.781179 & 1.151942 & -0.000001 \\
\hline 4 & 6 & 0 & 1.388420 & -0.100548 & -0.000002 \\
\hline 5 & 6 & 0 & 0.607104 & -1.252401 & -0.000002 \\
\hline 6 & 6 & 0 & -0.781230 & -1.151909 & 0.000006 \\
\hline 7 & 1 & 0 & -2.469274 & 0.178888 & 0.000009 \\
\hline 8 & 1 & 0 & -1.079698 & 2.227642 & 0.000013 \\
\hline 9 & 1 & 0 & 1.389383 & 2.048887 & 0.000028 \\
\hline 10 & 1 & 0 & 2.469279 & -0.178808 & 0.000009 \\
\hline 11 & 1 & 0 & 1.079631 & -2.227674 & -0.000007 \\
\hline 12 & 1 & 0 & -1.389318 & -2.048931 & 0.000000 \\
\hline
\end{tabular}




\section{Atom Coordinates and Absolute Energies (Calculated using Gaussian 03)}

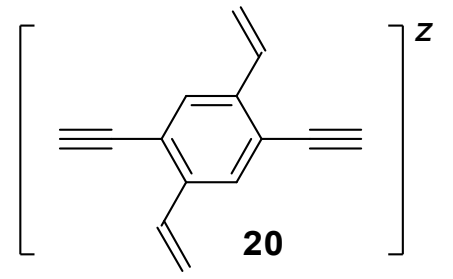

B3LYP/6-311++G(2d,p)

$z=0: \mathrm{HF}=-539.4944241$

$z=1: \mathrm{HF}=-539.2024989$ (same geometry used as obtained for neutral structure)

\begin{tabular}{|c|c|c|c|c|c|}
\hline \multirow{2}{*}{$\begin{array}{l}\text { Center } \\
\text { Number }\end{array}$} & \multirow{2}{*}{$\begin{array}{l}\text { Atomic } \\
\text { Number }\end{array}$} & \multirow{2}{*}{$\begin{array}{c}\text { Atomic } \\
\text { Type }\end{array}$} & \multicolumn{3}{|c|}{ Coordinates (Angstroms) } \\
\hline & & & $\mathrm{x}$ & $\mathrm{Y}$ & Z \\
\hline 1 & 6 & 0 & 1.134451 & 0.821221 & -0.000233 \\
\hline 2 & 6 & 0 & 1.300260 & -0.585095 & -0.063851 \\
\hline 3 & 6 & 0 & 0.148626 & -1.368931 & -0.068191 \\
\hline 4 & 6 & 0 & -1.134473 & -0.821211 & -0.000179 \\
\hline 5 & 6 & 0 & -1.300286 & 0.585089 & 0.063507 \\
\hline 6 & 6 & 0 & -0.148618 & 1.368922 & 0.067781 \\
\hline 7 & 6 & 0 & 2.256357 & 1.704046 & 0.015815 \\
\hline 8 & 6 & 0 & 3.185574 & 2.466908 & 0.029434 \\
\hline 9 & 6 & 0 & -2.256298 & -1.704137 & -0.016201 \\
\hline 10 & 6 & 0 & -3.185499 & -2.467026 & -0.029444 \\
\hline 11 & 6 & 0 & -2.643808 & 1.173678 & 0.126281 \\
\hline 12 & 6 & 0 & 2.643780 & -1.173747 & -0.126614 \\
\hline 13 & 6 & 0 & -2.965088 & 2.446895 & -0.107633 \\
\hline 14 & 6 & 0 & 2.965037 & -2.446622 & 0.109139 \\
\hline 15 & 1 & 0 & 0.237077 & -2.444431 & -0.142724 \\
\hline 16 & 1 & 0 & -0.237036 & 2.444432 & 0.142209 \\
\hline 17 & 1 & 0 & 4.008371 & 3.139899 & 0.044017 \\
\hline 18 & 1 & 0 & -4.008101 & -3.140260 & -0.043857 \\
\hline 19 & 1 & 0 & -3.439233 & 0.478782 & 0.373178 \\
\hline 20 & 1 & 0 & 3.439068 & -0.479252 & -0.375083 \\
\hline 21 & 1 & 0 & -3.992077 & 2.779601 & -0.027757 \\
\hline 22 & 1 & 0 & -2.235702 & 3.195037 & -0.394974 \\
\hline 23 & 1 & 0 & 3.991902 & -2.779666 & 0.029098 \\
\hline 24 & 1 & 0 & 2.235650 & -3.194094 & 0.398227 \\
\hline
\end{tabular}




\section{Atom Coordinates and Absolute Energies (Calculated using Gaussian 03)}<smiles>[Z]C#Cc1cc(=C)c(C#CC)cc1=C</smiles>

B3LYP/6-311++G(2d,p)

$z=0: \mathrm{HF}=-462.0437869$

$z=1: \mathrm{HF}=-461.7650869$ (same geometry used as obtained for neutral structure)

\begin{tabular}{|c|c|c|c|c|c|}
\hline \multirow{2}{*}{$\begin{array}{l}\text { Center } \\
\text { Number }\end{array}$} & \multirow{2}{*}{$\begin{array}{l}\text { Atomic } \\
\text { Number }\end{array}$} & \multirow{2}{*}{$\begin{array}{l}\text { Atomic } \\
\text { Type }\end{array}$} & \multicolumn{3}{|c|}{ Coordinates (Angstroms) } \\
\hline & & & $\mathrm{x}$ & Y & Z \\
\hline 1 & 6 & 0 & 1.404681 & -0.268118 & -0.000414 \\
\hline 2 & 6 & 0 & 0.914725 & 1.121459 & -0.000131 \\
\hline 3 & 6 & 0 & -0.524503 & 1.297859 & 0.000257 \\
\hline 4 & 6 & 0 & -1.404681 & 0.268120 & 0.000297 \\
\hline 5 & 6 & 0 & -0.914725 & -1.121458 & -0.000002 \\
\hline 6 & 6 & 0 & 0.524503 & -1.297858 & -0.000391 \\
\hline 7 & 6 & 0 & 2.807707 & -0.520276 & -0.000678 \\
\hline 8 & 6 & 0 & 3.992594 & -0.723489 & 0.000937 \\
\hline 9 & 6 & 0 & -2.807707 & 0.520277 & 0.000619 \\
\hline 10 & 6 & 0 & -3.992595 & 0.723483 & -0.000368 \\
\hline 11 & 6 & 0 & -1.740386 & -2.187573 & 0.000067 \\
\hline 12 & 6 & 0 & 1.740387 & 2.187575 & -0.000227 \\
\hline 13 & 1 & 0 & -0.900181 & 2.313945 & 0.000507 \\
\hline 14 & 1 & 0 & 0.900181 & -2.313943 & -0.000642 \\
\hline 15 & 1 & 0 & 5.038736 & -0.912116 & 0.000906 \\
\hline 16 & 1 & 0 & -5.038737 & 0.912109 & -0.000227 \\
\hline 17 & 1 & 0 & -2.815341 & -2.071080 & 0.000340 \\
\hline 18 & 1 & 0 & 2.815342 & 2.071082 & -0.000501 \\
\hline 19 & 1 & 0 & -1.342937 & -3.194561 & -0.000130 \\
\hline 20 & 1 & 0 & 1.342937 & 3.194562 & -0.000049 \\
\hline
\end{tabular}

